Revue des patrimoines

32 | 2017

Le collectif à l'œuvre. Collaborations entre architectes et plasticiens (XXe-XXle siècles)

\title{
Guy Rottier, un architecte-artiste parmi les artistes
}

Guy Rottier, an artist-architect amongst artists

\section{Nouha Babay}

\section{(2) OpenEdition}

\section{Journals}

Édition électronique

URL : http://journals.openedition.org/insitu/15281

DOI : 10.4000/insitu. 15281

ISSN : $1630-7305$

Éditeur

Ministère de la culture

Référence électronique

Nouha Babay, «Guy Rottier, un architecte-artiste parmi les artistes », In Situ [En ligne], 32 | 2017, mis en ligne le 24 juillet 2017, consulté le 19 avril 2019. URL : http://journals.openedition.org/insitu/15281 ; DOI : 10.4000/insitu.15281

Ce document a été généré automatiquement le 19 avril 2019

\section{cc) (†)}

In Situ Revues des patrimoines est mis à disposition selon les termes de la licence Creative Commons Attribution - Pas d'Utilisation Commerciale - Pas de Modification 4.0 International. 


\title{
Guy Rottier, un architecte-artiste parmi les artistes
}

\author{
Guy Rottier, an artist-architect amongst artists
}

\section{Nouha Babay}

1 L'architecte franco-néerlandais Guy Rottier (1922-2013) a placé la rencontre et la collaboration entre architectes et artistes au cœur de sa démarche. Disciple de Le Corbusier, diplômé de l'École des beaux-arts en 1952, il n'a eu de cesse de dépasser les frontières entre art et architecture. Seul architecte du groupe des artistes de l'École de Nice, membre du Groupe international d'architecture prospective (GIAP) et fondateur du groupe des « Conspiratifs », ses recherches témoignent des collaborations fructueuses qui peuvent naître entre architectes et artistes. Au-delà du fait qu'elle s'inscrit souvent dans une dynamique collaborative, la pratique personnelle de l'architecte possède une dimension transdisciplinaire intrinsèque. En 1964, Guy Rottier intègre en effet le groupe des artistes de l'École de Nice. Cette rencontre marque une rupture significative dans sa carrière, le faisant passer d'une activité purement architecturale à une pratique plastique de l'architecture. L'objectif de cet article est de revenir sur cette expérience pour comprendre ce basculement dans la démarche de Guy Rottier et pour questionner les modalités de collaboration entre artistes et architectes. L'étude de quelques projets, certains restés théoriques et d'autres ayant donné lieu à des réalisations, permet de poser quelques jalons quant au cheminement de Guy Rottier vers l'«arTchitecture », concept qu'il formule dès 1987. L'enjeu est de comprendre dans quelle mesure l'architecture devient pour lui synonyme d'une expérience globale. Enfin, à travers son expérience au sein du GIAP et des "Conspiratifs », on mesurera l'importance du collectif comme moteur de création individuelle.

\section{Le mythe des origines}

2 Né à Tanjung Morawa (Sumatra, Indonésie) le 16 juillet 1922, l'architecte et l'ingénieur Willem Frederik Henri Rottier, dit Guy Rottier (fig. 1), est considéré comme l'une des 
figures pionnières de la prospective architecturale ${ }^{1}$. Sa jeunesse placée sous le signe de la multi-culturalité, sa fréquentation des artistes et son parcours atypique vont lui permettre de formuler des hypothèses pour le moins insolites quant à l'architecture et la ville du futur. En effet, après avoir fréquenté tour à tour l'école primaire hollandaise de Sumatra, l'école primaire de Soest aux Pays-Bas, Guy Rottier arrive à Grasse (AlpesMaritimes) à l'âge de neuf ans. En septembre 1943, alors qu'il s'apprête à terminer sa deuxième partie du baccalauréat en mathématiques élémentaires pour rejoindre l'École des beaux-arts de Paris en section peinture, il doit quitter la France sous la pression des troupes d'occupation italiennes pour rejoindre La Haye (Pays-Bas). Malgré sa volonté de devenir peintre, il renonce à ce projet sur les conseils de son père, ingénieur agronome, et intègre le Technikum de La Haye (ancien Middelbare Technische School) ${ }^{2}$ d'abord en section design, puis en section bâtiment. Comme beaucoup de ses contemporains, sa scolarité est fortement contrariée par la guerre et, en juillet 1944, il interrompt ses études, contraint de rejoindre le Service du travail obligatoire (STO) où il travaille comme dessinateur sur le projet de l'usine souterraine Carl Zeiss ${ }^{3}$ dans la ville d'Iéna en Allemagne $e^{4}$.

Figure 1

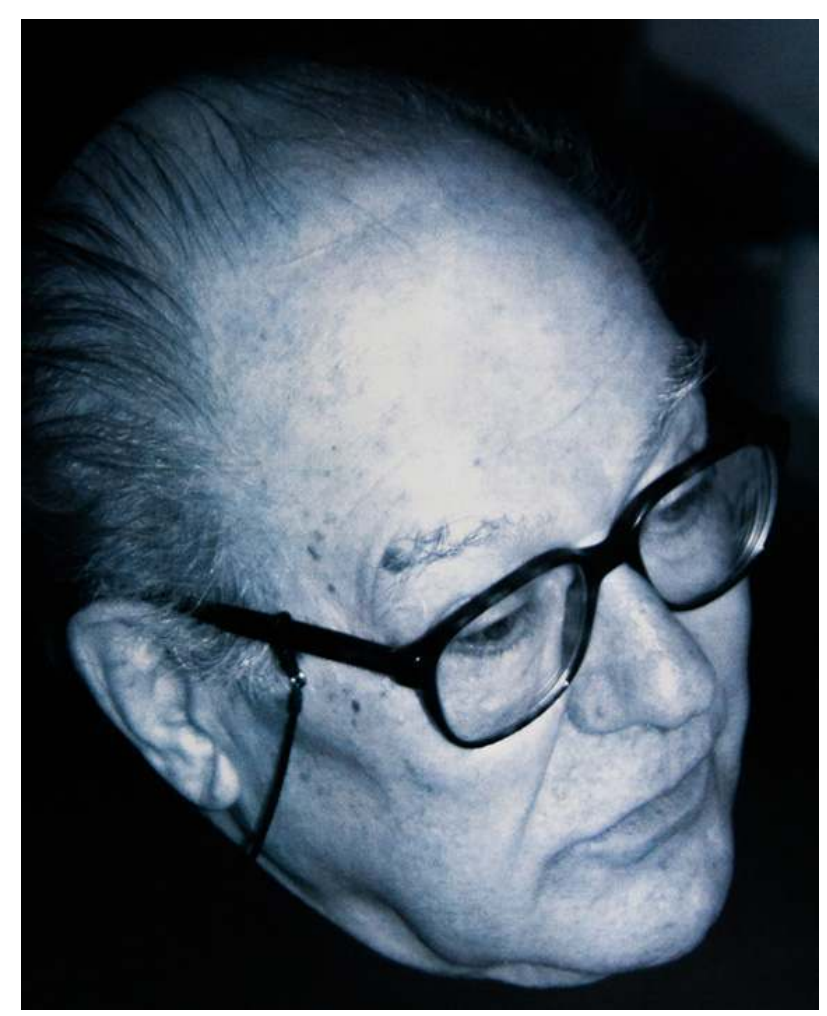

PORTRAIT DE GUY ROTTIER, 2006.

(C) ARCHIVES PRIVÉES ODETTE BARBERIS-ROTTIER, 2014.

Libéré par les Américains en 1944, Guy Rottier obtient son diplôme d'ingénieur en bâtiment en décembre 1945. Se considérant plus Français que Néerlandais et déterminé à obtenir le diplôme d'architecte délivré par l'École des beaux-arts de Paris, il travaille comme traducteur français-néerlandais à l'ambassade des Pays-Bas à Paris. Cet emploi lui permet d'obtenir, en janvier 1947, un logement dans le pavillon néerlandais à la Cité internationale universitaire où il est confronté in situ à un éclectisme architectural et 
culturel novateur et formateur ${ }^{5}$. Par ailleurs, il réussit le concours d'admission de l'École des beaux-arts de Paris le 7 juillet 1947 et intègre la deuxième classe de la section architecture, suivant notamment les cours du soir des ateliers Henri Madelain-Guillaume Gillet et André Lurçat ${ }^{6}$. Cette immersion culturelle lui permet d'être au contact avec l'actualité parisienne. Il réussit même à rencontrer André Wogensky ${ }^{7}$ - collaborateur de Le Corbusier - lors d'une conférence sur l'Unité d'habitation de Marseille (1947-1952) perçue à l'époque comme un véritable manifeste architectural concrétisant le projet de cité radieuse imaginé par Le Corbusier en 1934. Cette rencontre le détermine à rejoindre l'agence de la rue de Sèvres qu'il intègre en tant que dessinateur (fig. 2) puis de conducteur de travaux à l'Unité d'habitation de Marseille (fig. 3).

Figure 2

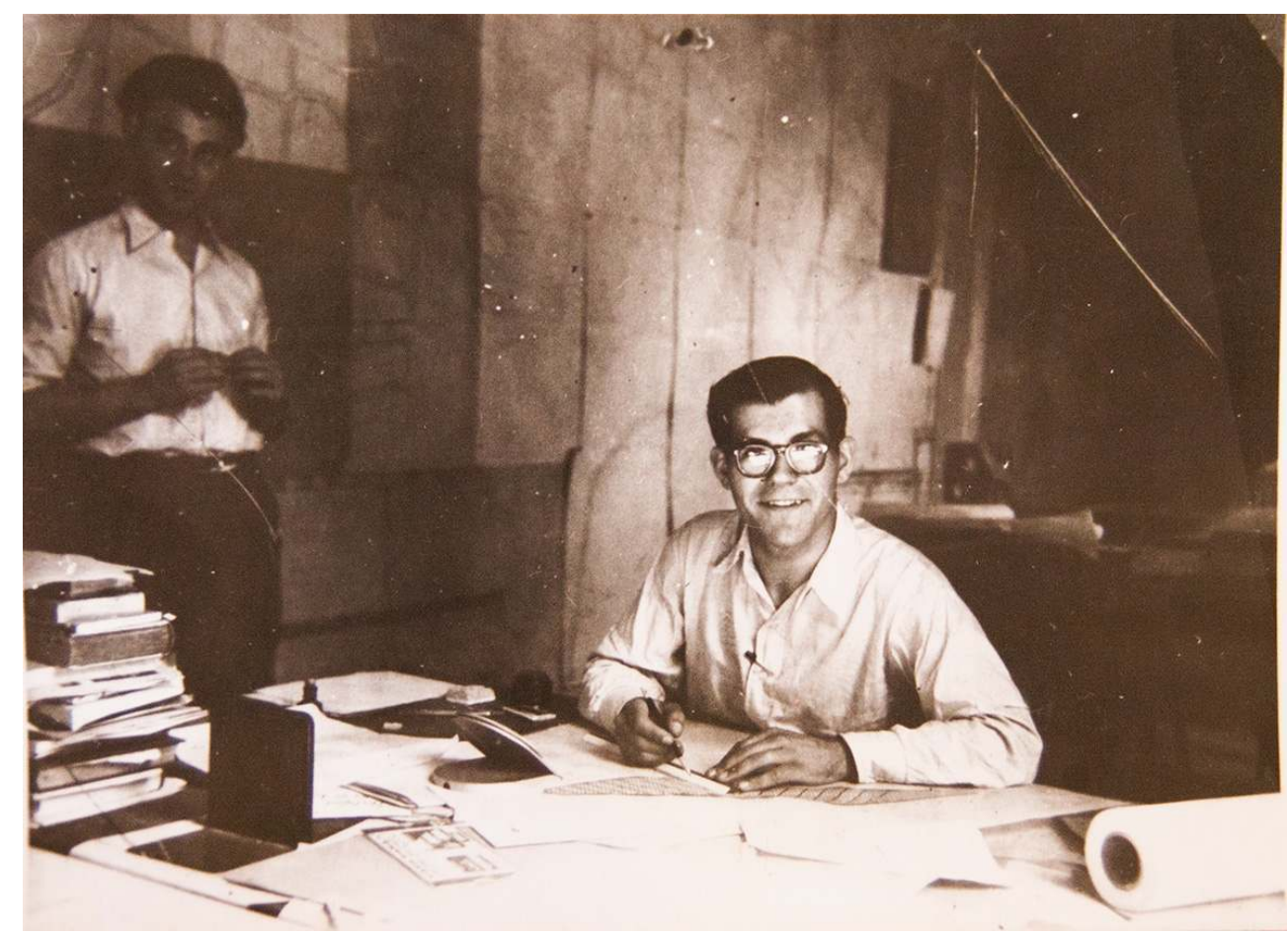

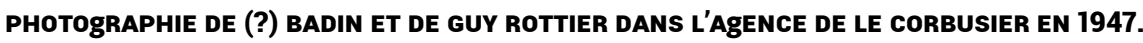

(C) ARCHIVES PRIVÉES ODETTE BARBERIS-ROTTIER, 2014. 


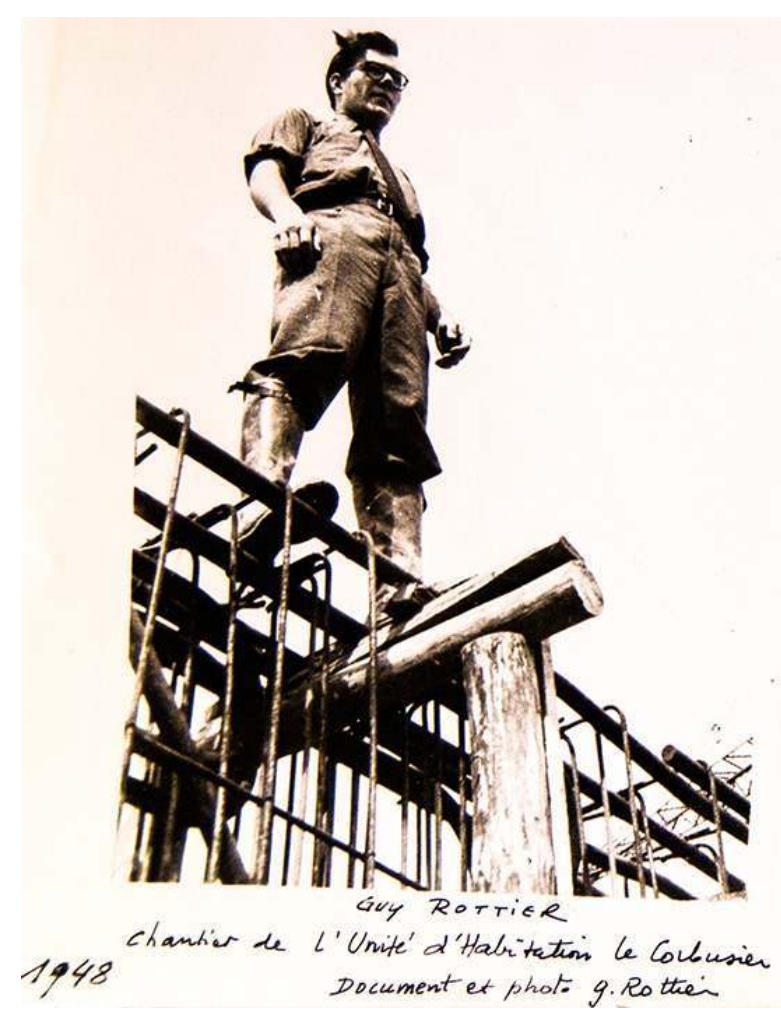

PHOTOgRAPHIE DE GUY ROTTIER LORS DU FERRAILLAgE DU SOL ARTIFICIEL DE L'UNITÉ D'HABITATION DE MARSEILLE EN 1948.

(C) ARCHIVES PRIVÉES ODETTE BARBERIS-ROTTIER, 2014.

4 Travailler dans l'agence de Le Corbusier, de surcroît sur ce projet ${ }^{8}$, permet à Guy Rottier de rencontrer des architectes et artistes de sa génération (Roger Aujame, Georges Candilis, Shadrach Woods, Pirkko Kaarina Hirvelä, Márta Pán, Pierre Rosenberg, Nikos Chátzidakis, Fernand Gardien et Roggio Andreini notamment), mais aussi les ingénieurs (Ernst Neufert et Jean Prouvé), l'artiste Pablo Picasso ou encore Charles Barberis ${ }^{9}$ (fig. 4). Ces rencontres marquent durablement Guy Rottier qui cherchera, tout au long de sa carrière, à retrouver l'esprit d'équipe, le multiculturalisme et l'effervescence qui caractérisaient l'agence Le Corbusier. D'autant que Guy Rottier commence à y croiser les disciplines ce qui, dans son esprit, contribue à estomper les limites entre art et architecture, même si cette démarche ne trouve tout son sens qu'au début des années 1960, au moment où il se rapproche des artistes de l'École de Nice. 
Figure 4

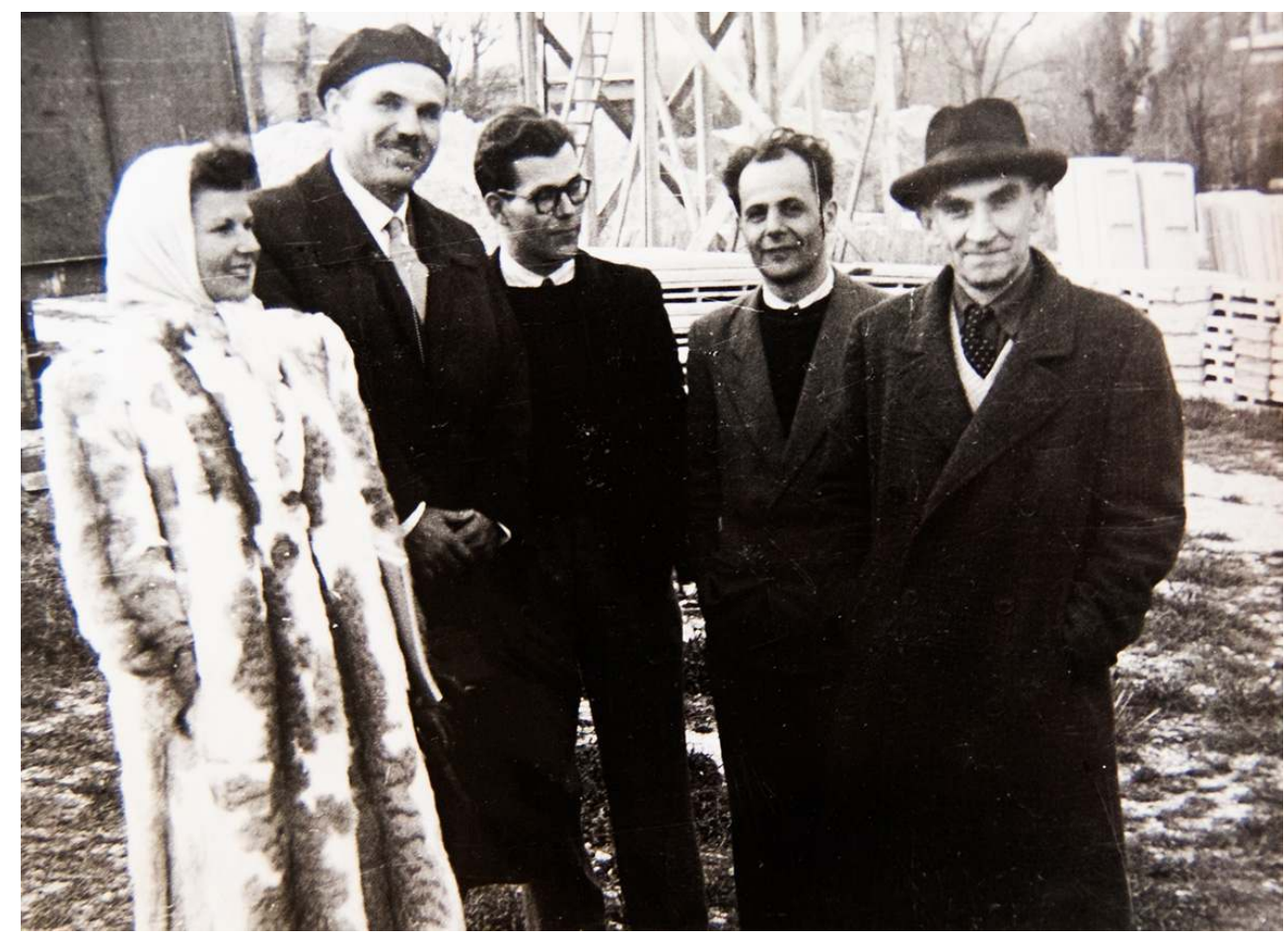

ERNEST NEUFERT, SON ÉPOUSE, GUY ROTTIER (AU CENTRE), GEORgES CANDILIS ET VLADIMIR BODIANSKY SUR LE CHANTIER DE L'UNITÉ D'HABITATION DE MARSEILLE EN 1949.

(C) ARCHIVES PRIVÉES ODETTE BARBERIS-ROTTIER, 2014.

\section{L'École de Nice, complicité et transgressions disciplinaires}




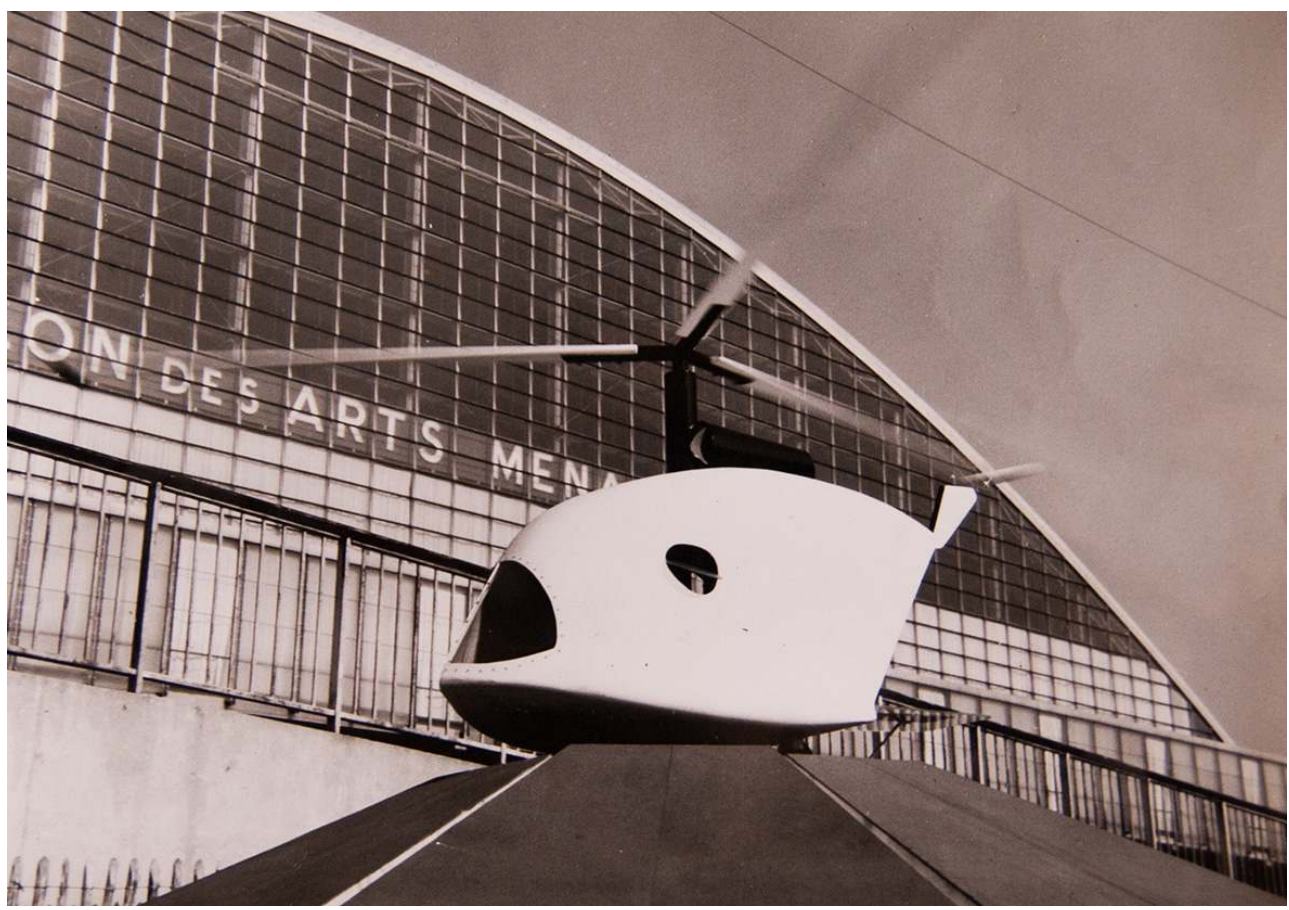

Photographie de la maquette (éch. 1/5) de la Maison de vacances (projet théorique, 1964) volante exposée au CNIT en 1964.

C C ARCHIVES PRIVÉES ODETTE BARBERIS-ROTTIER, 2014.

5 Alors que Guy Rottier a regagné le sud de la France depuis 1958, un événement majeur précipite sa carrière et le fait sortir de son relatif isolement. Il s'agit de l'exposition en 1964, au Salon des arts ménagers qui se déroule au CNIT à La Défense, de son projet quelque peu provocateur de Maison volante (fig. 5). Cette médiatisation intervient en réalité à un moment difficile de la carrière du jeune architecte, à savoir l'échec d'habitats de vacances industrialisés qu'il avait conçus en 1958 avec Charles Barberis, le «menuisier» de Le Corbusier. Se heurtant à la réticence des services administratifs qui refusent d'homologuer ces cabanons destinés à être commercialisés sur catalogue, Guy Rottier crée, en réaction face à une décision qu'il juge sclérosante, une Maison volante, audacieuse maison mobile qui prend la forme d'un hélicoptère. Ce projet obtient une certaine audience dans la presse nationale et internationale ${ }^{10}$ et contribue à le faire connaître. En 1965, un jeune artiste, Bernar Venet, rend visite à Guy Rottier dans son agence à Nice $^{11}$ pour échanger autour de la Maison volante mais aussi des maisons enterrées imaginées par Rottier depuis 1964. Bernar Venet s'est pour sa part fait récemment connaître avec Tas de charbon (1963), sculpture sans forme spécifique composée d'un tas de charbon versé à même le sol qui lui avait été inspirée par la découverte fortuite, alors qu'il était assistant-décorateur à l'opéra de Nice (1958-1960), d'une route où un chargement de goudron avait été déversé. La solidification spontanée de cette matière avait attiré son attention et il réalise dès 1960 une série de peintures recouvertes de goudron ${ }^{12}$. Une connivence s'établit spontanément entre les deux hommes, du fait de la proximité de leurs démarches. Si dans le cas de Venet l'objectif est de libérer la forme qui, par une action poïétique, crée une sculpture se déployant «naturellement» dans l'espace d'exposition, pour Guy Rottier, il s'agit de créer des formes fonctionnelles destinées à être habitées. La Maison industrialisée enterrée qu'il 
projette en 1964 est une structure préfabriquée assemblée sur site et recouverte de terre (donc enterrée a posteriori). C'est une maison dépourvue de façade qui, selon l'architecte, constitue une manière d'habiter le paysage sans l'altérer. En plus d'imaginer des architectures à l'apparence de "sculptures végétales» dans lesquelles domine la géométrie, Guy Rottier propose de couvrir de manière aléatoire les maisons avec des matériaux usagés récupérés sur site ou dans les déchetteries ${ }^{13}$ (fig. 6). De cette manière, il pense participer à la constitution d'un paysage spontané, au même titre que Bernar Venet au travers de l'œuvre Tas de Charbon.

Figure 6

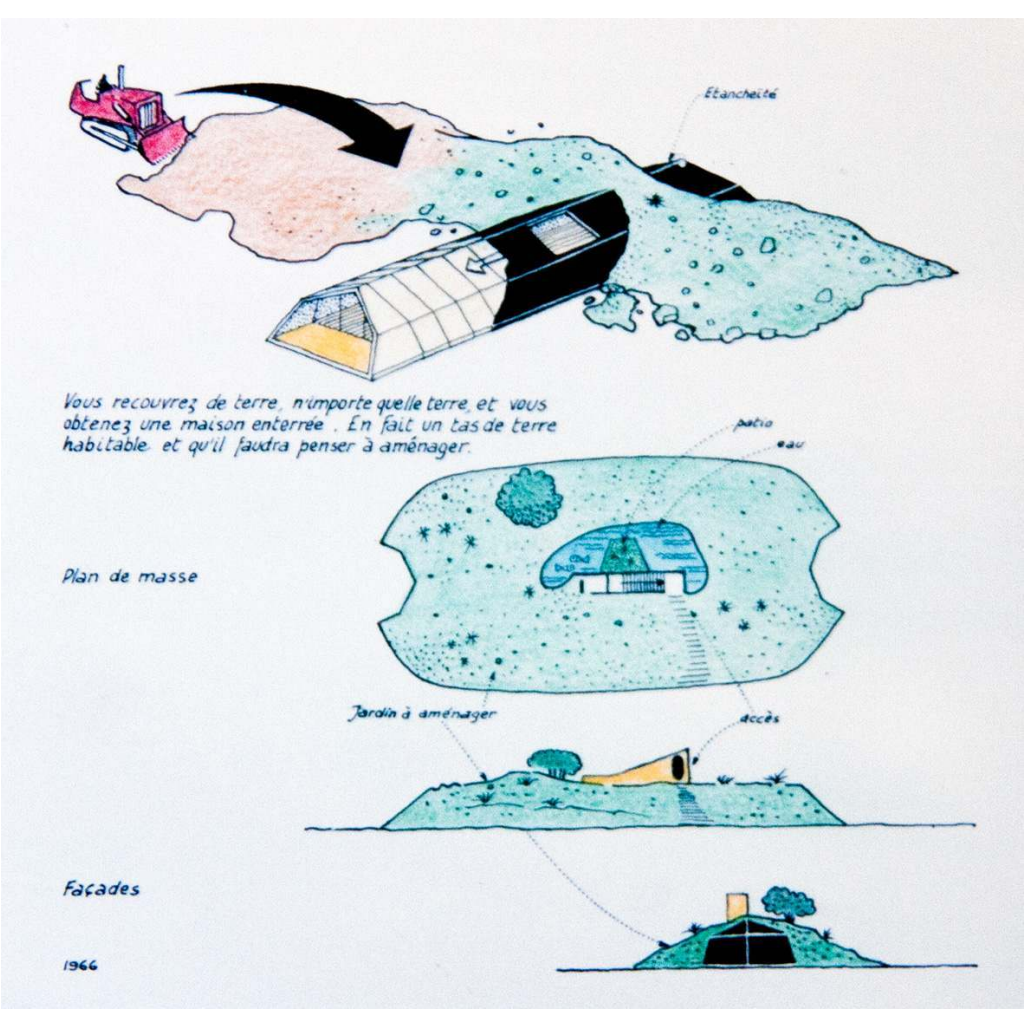

MAISON INDUSTRIALISÉE ENTERRÉE (PROJET THÉORIQUE, 1965).

REPRO. NOUHA BABAY, 2014. (C ARCHIVES PRIVÉES ODETTE BARBERIS-ROTTIER.

Par l'intermédiaire de Bernar Venet, Guy Rottier se rapproche des artistes de l'École de Nice: Fernandez Arman (dit Arman), Claude Gilli, Claude Farhi, Marcel Alloco, Ben Vautier, Albert Chubac, Noël Dolla, Claude Viallat. Comme eux, Guy Rottier est à la recherche de nouveaux moyens d'expression. L'aventure collective commence au café Félix-Faure à Nice et dans la boutique de brocante de Ben Vautier où de jeunes artistes diplômés de l'École des arts décoratifs de $\mathrm{Nice}^{14}$, autodidactes ou issus de divers mouvements ou tendances ${ }^{15}$, se retrouvent pour échanger. Ils ont en commun de vouloir faire émerger une synergie créatrice hors de tout cadre officiel et en rupture avec l'abstraction lyrique de l'École de Paris. Si leur ambition est de s'inscrire pleinement dans une société en pleine mutation, l'École de Nice sera aussi «une affaire d'amitiés, de brouilles et de réconciliations ${ }^{16}{ }^{1}$ (fig. 7, fig. 8). Bien que géographiquement éloigné des centres artistiques majeurs qui sont alors Paris et New York, le groupe niçois se pose dans la continuité des pratiques de ready-made initiées par Marcel Duchamp ${ }^{17}$ et du Nouveau Réalisme d'Yves Klein, Fernandez Arman et Martial Raysse. Le courant européen Fluxus ${ }^{18}$, 
par la variété des champs d'expression artistique qu'il embrasse (arts plastiques, théâtre, musique, littérature), nourrit aussi cette pépinière d'idées par l'intermédiaire de Marcel Alocco et de Ben Vautier. Enfin, Claude Viallat, enseignant à l'école des Arts décoratifs de Nice, y introduit le courant Supports-Surfaces. Ainsi, pendant une vingtaine d'années, Nice polarise une dynamique de création collective et stimulante à laquelle critiques d'art et galeristes contribuent à donner un cadre. D'ailleurs, le mouvement trouve son nom grâce à Pierre Restany et à Alexandre de La Salle qui organisent le 6 mars 1967 une rétrospective à l'occasion des " 10 ans d'activités des artistes de l'École de Nice $»^{19}$. Ils imposent une expression utilisée pour la première fois en 1960 par la critique Claude Rivière $^{20}$. L'importance du mouvement tient également à la notoriété internationale acquise par certains artistes, notamment Arman qui s'installe à New York dès $1961^{21}$. Les accumulations d'Arman, les patchworks de Marcel Alocco, les inscriptions de Ben Vautier, les sculptures de Bernar Venet, les objets Pop de Claude Gilli mais aussi les projets d'architecture de Guy Rottier vont ainsi être mis en regard dans des expositions collectives $^{22}$ dont la première se déroule à la galerie Knoll International (Paris ?) dirigée par le designer Raymond Cornillon en $1967^{23}$.

Figure 7

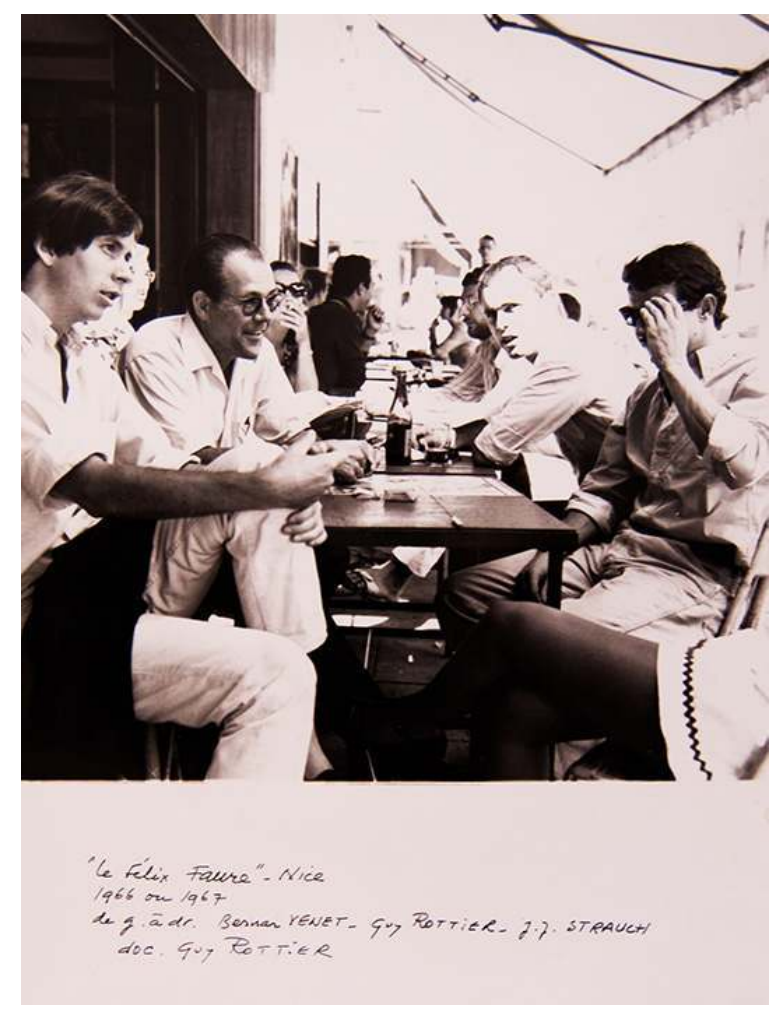

Rencontres des artistes de l'École de Nice au café Félix-Faure à Nice (s.d. circa 1966).

PHOT. J.-J. STRAUCH, 2014. @ ARCHIVES PRIVÉES OdETTE BARBERIS-ROTTIER. 
Figure 8

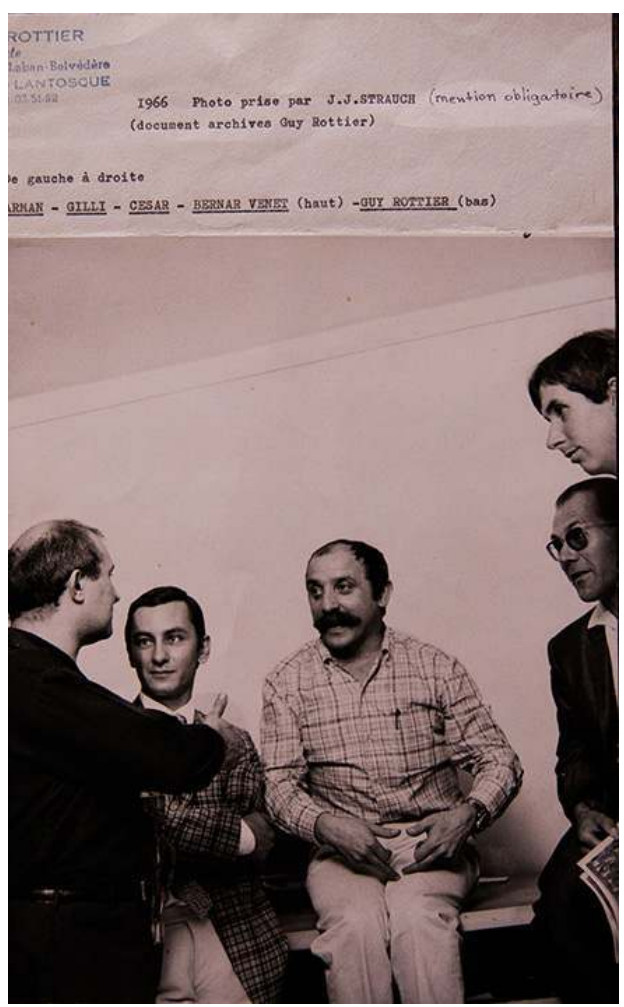

GUY ROTTIER, FERNANDEZ ARMAN, CLAUDE GILLI, CÉSAR BALDACCINI ET BERNAR VENET EN 1966.

PHOT. J.-J. STRAUCH, 2014. ( ARCHIVES PRIVÉES ODETTE BARBERIS-ROTTIER.

Guy Rottier est le seul architecte à intégrer le groupe de plasticiens. Cela tient à sa volonté personnelle d'inscrire son action dans une dynamique de synthèse des arts d'ascendance corbuséenne ${ }^{24}$. Cette immersion dans une " communauté » de créateurs lui permet surtout d'exprimer des idées souvent hétérodoxes, d'exposer des projets insolites, en somme elle offre à l'architecte un espace de liberté qui lui permet de s'abstraire de toute contrainte administrative et constructive. Cette proximité a laissé des traces dans les archives de Guy Rottier : œuvres offertes ou reçues, lettres et mots échangés mais aussi et surtout des projets d'architecture que Guy Rottier dédie ou réalise à l'attention de l'un ou de l'autre. Si dans la Maison de Farhi (projet théorique, 1967) lieu de vie et atelier s'intégraient dans le paysage, la Maison enterrée pour Ben (projet théorique, 1967) est couverte de carcasses de voitures faisant référence aux accumulations d'Arman et à la Compression plate (1970) de César Baldaccini. La Maison solaire (projet théorique, 1982) que Guy Rottier imagine à nouveau pour Ben en 1982 est pour sa part une déclinaison de la Maison lumière (projet théorique, 1973) où il recourait à l'« enveloppe caméléon » qu'il avait élaborée en 1974. En 1989, Guy Rottier imagine même un musée dédié aux artistes de l'École de Nice, mobilisant tous les concepts architecturaux qui lui sont chers enfouissement, solaire, gonflable - et scénographie particulière (fig. 9). 


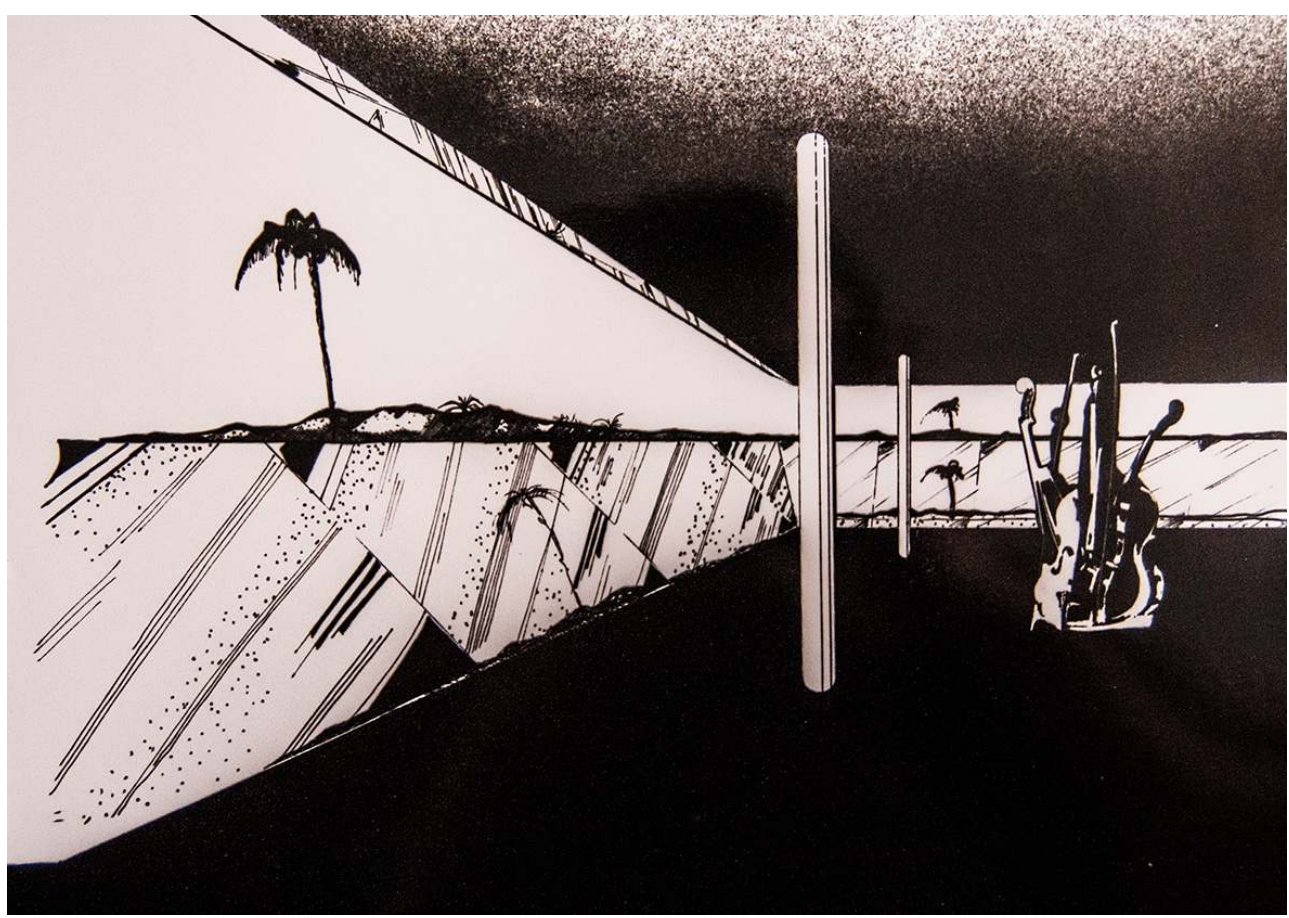

MUSÉE POUR L'ÉCOLE de NICE (PROJET THÉorIQUe, 1989) : VUE EN PERSPECTIVE SUR LA SALLE de PÉRISCOPES, ENCRE, STYLOS FEUTRES ET PEINTURE SUR PAPIER, 50 x 65 cM).

(c) FONDS GUY ROTTIER, FRAC ORLÉANS, DÉPÔT GUY ROTTIER, NON COTÉ.

D'autres projets sont conçus en binôme, comme par exemple Architecture "camouflage » (1969), procédé photographique par lequel Guy Rottier et Bernar Venet imaginent des façades interchangeables selon la nature environnante. Quand Marcel Alocco conçoit son Musée sans architecte (1967) (fig. 10), il se nourrit de ses échanges avec Guy Rottier qui considère notamment que "pour apprécier réellement une exposition, il faut avoir le nez dedans $»^{25}$. Les échanges sont si nombreux qu'ils constituent l'un des fondements de ce collectif qui aspire à dépasser les dogmes. En 1967, Arman, qui bénéficie déjà d'une certaine renommée, confie à Guy Rottier la conception de sa maison de vacances à Vence (fig. 11). 
Figure 10

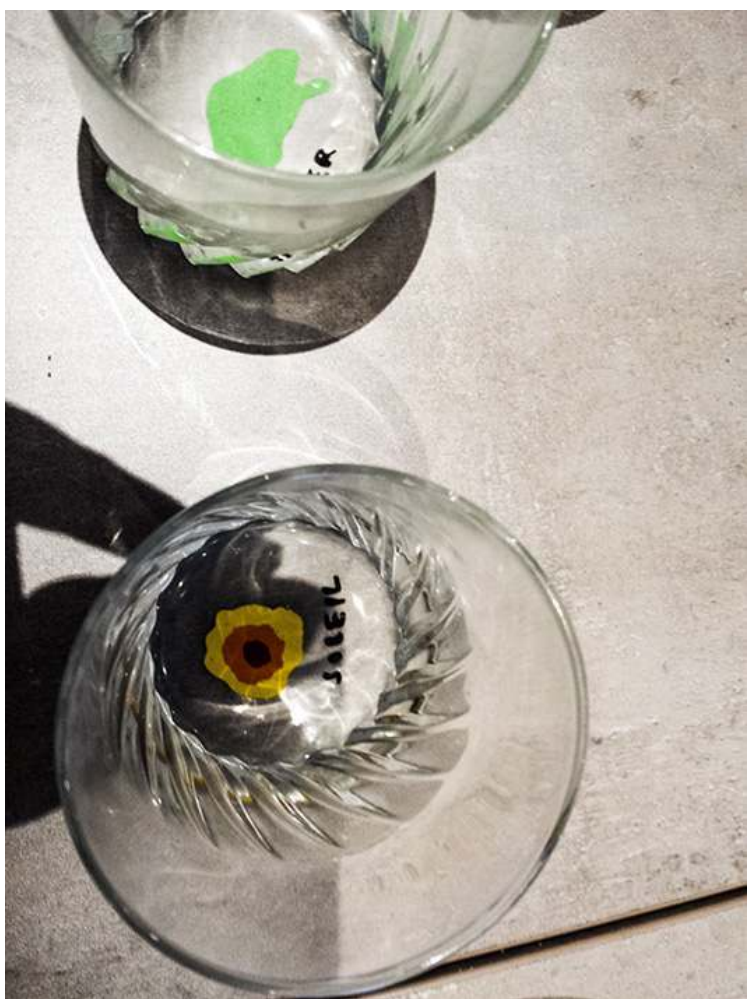

MUSÉE SANS ARCHITECTE (EN COLLABORATION AVEC MARCEL ALLOCO, 1989), INSTALLATION (VERRE ET PEINTURE).

(C) ARCHIVES PRIVÉES DE MARCEL ALLOCO. 


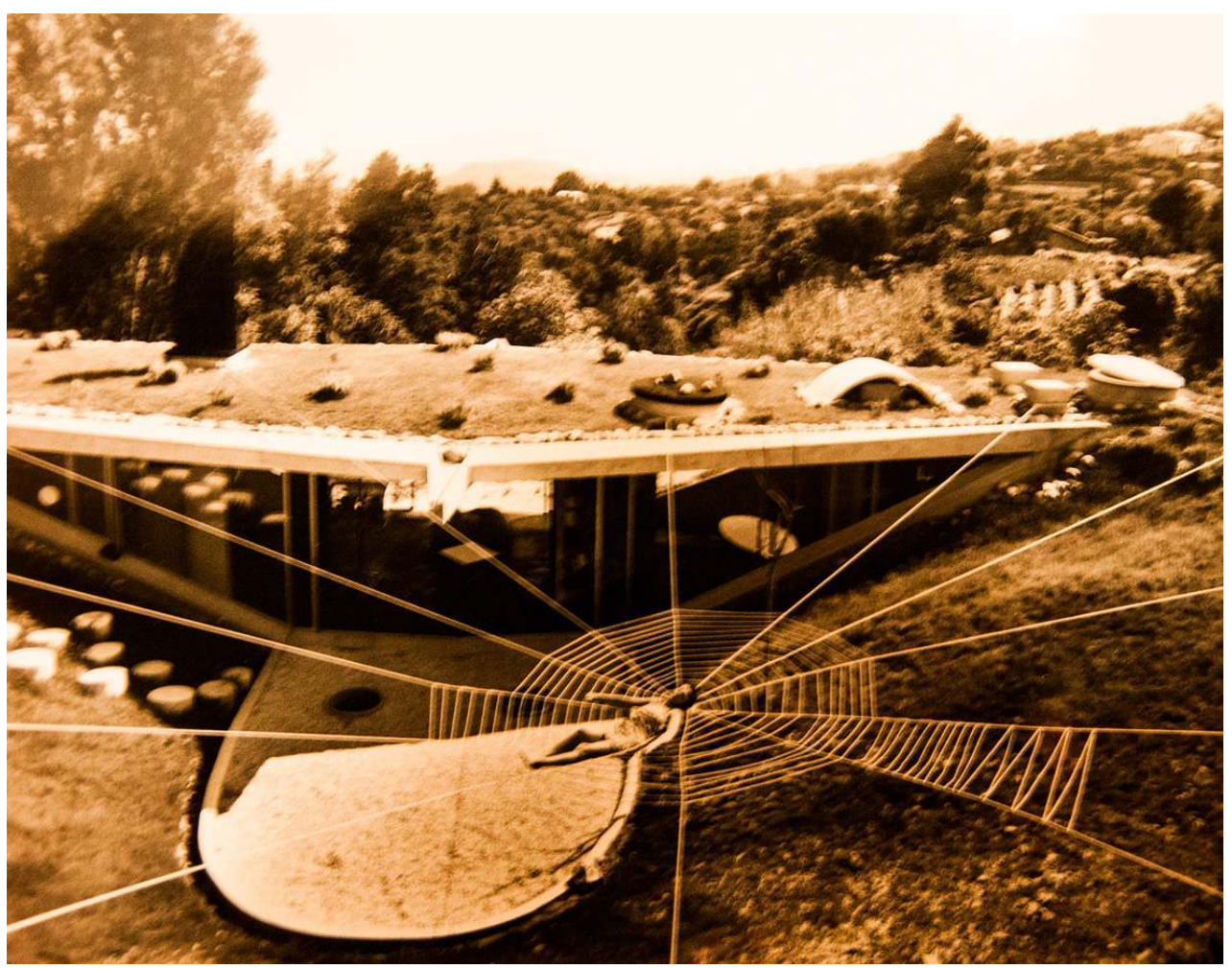

VUE DE LA PARTIE NORD DE LA VILLA ARMAN EN 1968.

PHOT. GUY ROTTIER. (C) ARCHIVES PRIVÉES ODETTE BARBERIS-ROTTIER.

La villa Arman, construite en 1968, représente un manifeste car elle est l'occasion pour son auteur de mettre en pratique certaines de ses idées les plus audacieuses, notamment pour les dispositifs semi-enterrés et mobiles. Elle témoigne aussi des prolongements, parfois transgressifs, à l'œuvre dans le collectif car l'artiste l'investit progressivement par ses accumulations. Par ce processus d'appropriation, il estompe le caractère originel de la demeure et la fait glisser vers une autre forme d'expression artistique. L'architecture de Guy Rottier devient ainsi une œuvre sculpturale d'Arman (fig. 12), questionnant par là même les liens entre art et architecture. Alors que l'architecte souhaitait mettre à profit la liberté laissée par l'artiste pour introduire une dimension esthétique et plastique dans sa production, l'artiste a convoité l'investissement de l'espace, envahissant, se réappropriant et détournant le cadre bâti. C'est certainement ainsi que peuvent naître des formes de création hybrides que Martine Bouchier définit comme un « antagonisme réciproque et fondamental, une structure d'attraction-répulsion conditionnant? les opérations esthétiques et productrices de ce que l'on peut appeler la relation art(s)architecture $»^{26}$. 


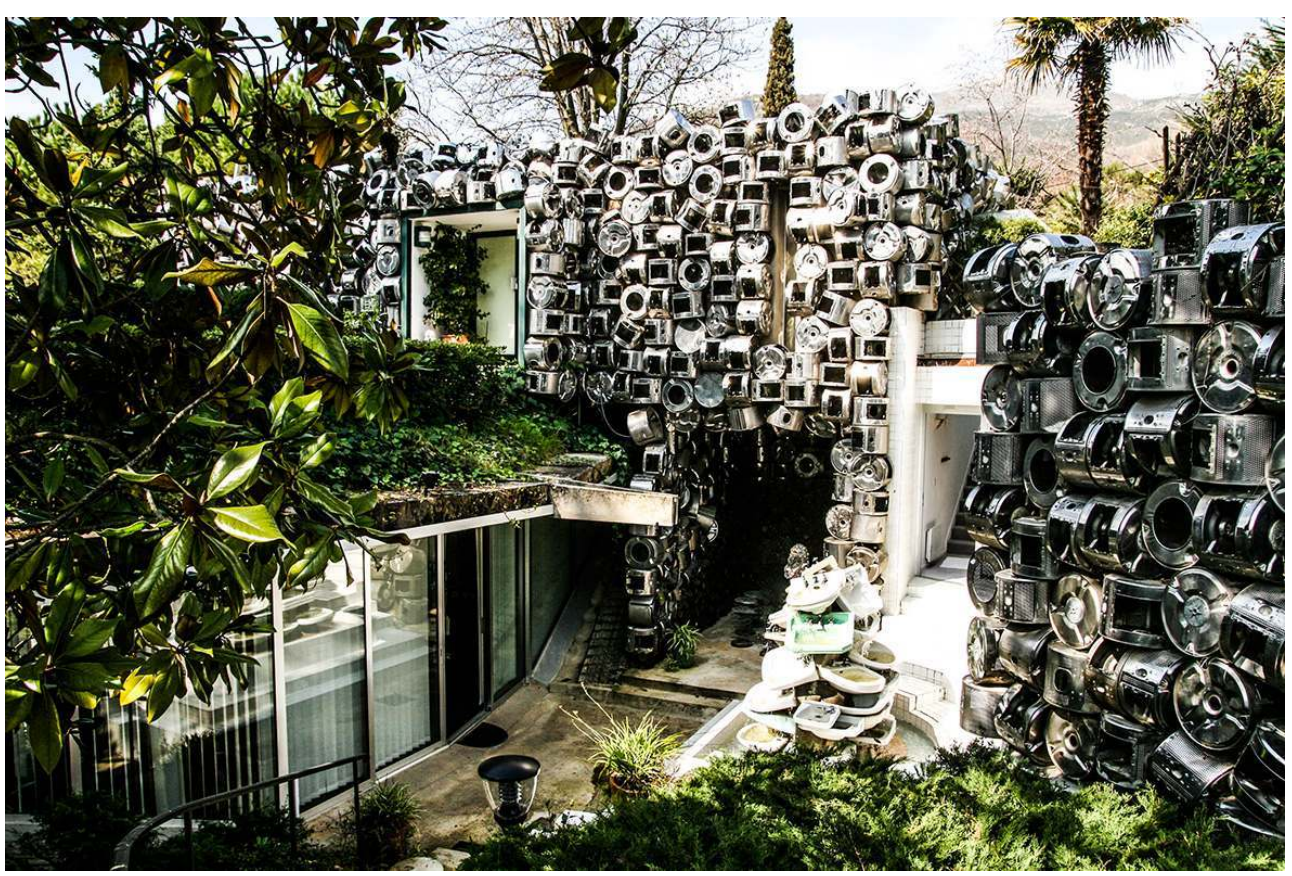

VUE de LA PARTIE NORd DE LA VILLA ARMAN EN 2014 : UNe CHAMBre A ÉTÉ CONSTRUITE AU débUt DES ANNÉES 1980 et LA VILLA A ÉTÉ COUVERTE PAR DES TAMBOURS DE MACHINE À LAVER PAR ARMAN.

PHOT. NOUHA BABAY. () NOUHA BABAY.

\section{Le Groupe international d'architecture prospective (GIAP), un autre élan collectif pour Guy Rottier}

10 Parallèlement à son compagnonnage avec les artistes de l'École de Nice, dès 1965, Guy Rottier intègre le GIAP. Ce groupe, créé officiellement en mai $1965^{27}$ sous la houlette de l'historien et critique d'art et d'architecture Michel Ragon ${ }^{28}$, réunit de manière plus ou moins formelle les architectes dit "prospectifs" (fig. 13). Le premier Festival de l'art d'avant-garde, qui se tient en 1956 sur le toit-terrasse de l'Unité d'habitation de Marseille, marque un moment fort dans le cheminement intellectuel de Michel Ragon, une sorte de "révélation» au cours de laquelle «le jeune critique d'art est confronté in situ à l'architecture de Le Corbusier $»^{29}$. Il publie ensuite son premier ouvrage sur l'architecture - Le Livre sur l'architecture moderne $e^{30}$ (1958) - puis, à partir de 1961, une série d'articles sur l'actualité architecturale dans la revue Arts10. Michel Ragon joue désormais un rôle important sur le plan intellectuel. Son engagement passe aussi par le collectif, au sein du Groupe des études architecturales mobiles (GEAM), formé en 1958, suite au dixième Congrès international d'architecture moderne (CIAM) qui s'était tenu à Dubrovnik, en Yougoslavie, sur le thème de l'architecture mobile. Guy Rottier y avait participé avec Charles Péré-Lahaille en proposant une cité ouvrière mobile ${ }^{31}$. La revue allemande Bauwelt consacre dès 1958 des articles au GEAM et Guy Rottier y publie quelques articles sur ses architectures mobiles ${ }^{32}$. Toutefois, il faut attendre l'exposition «Demain...Paris », organisée en 1961 par le ministère de la Construction au Grand Palais, pour que le grand public découvre les propositions de Ville spatiale de Yona Friedman ${ }^{33}$ et de Ville flottante en forme de pyramide de Paul Maymont ${ }^{34}$. Parallèlement, Michel Ragon théorise le concept 
d'architecture-sculpture ${ }^{35}$ et appelle à rapprocher rationalité et fonctionnalité dans des pratiques symboliques et poétiques. Ses textes servent de matrice théorique aux jeunes architectes, sociologues, artistes et intellectuels réunis au sein du GIAP pour réfléchir à la ville du futur et pour établir un lien entre le monde de l'art et celui de l'architecture. Plus largement, les écrits de Michel Ragon favorisent l'émergence d'alternatives architecturales et urbaines reposant sur les notions de prospective et futurologie. D'ailleurs, en 1963, Michel Ragon publie Où vivrons-nous demain ? ${ }^{36}$ qui, comme le souligne Hélène Jannière, « est presque entièrement dédié à l'exposition des projets prospectifs des architectes du futur GIAP $»^{37}$.

Figure 13

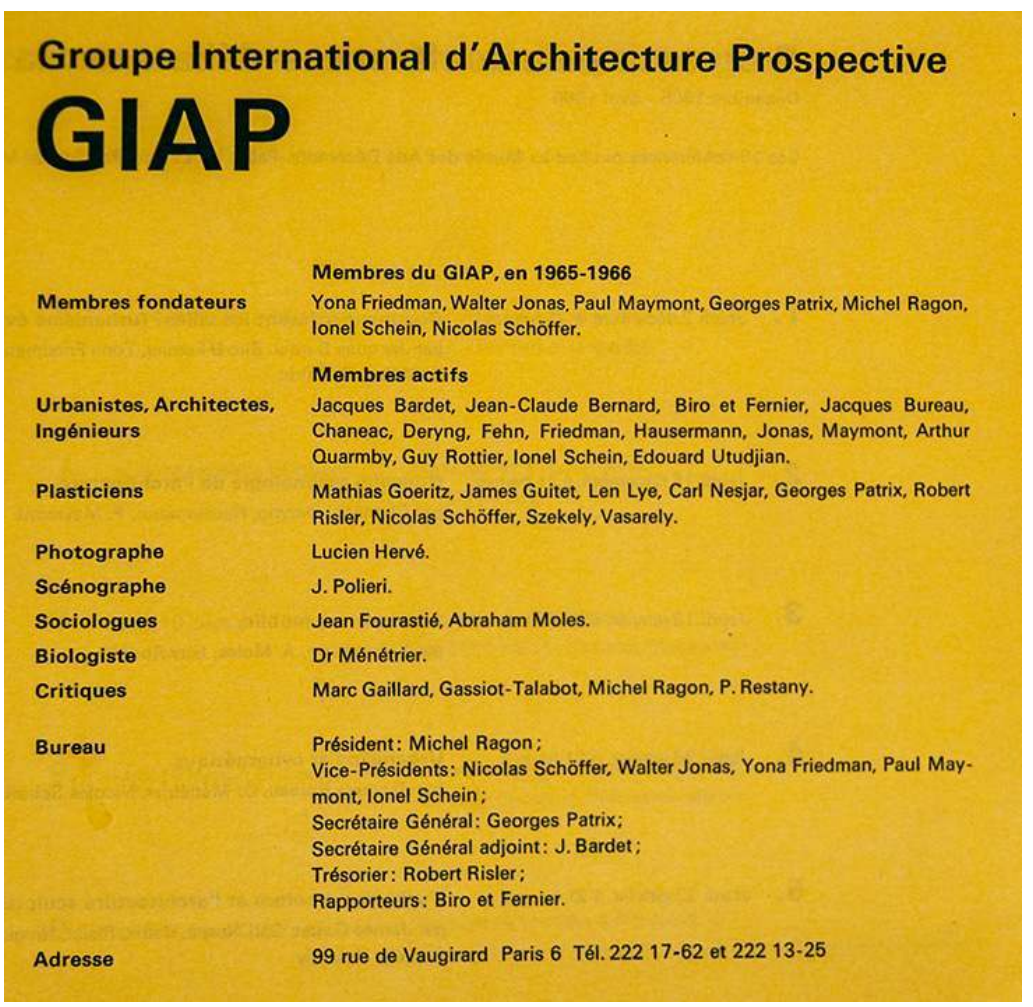

MANIFESTE DU GIAP (1964).

(c) ARCHIVES PRIVÉES ODETTE BARBERIS-ROTTIER.

11 Ces réflexions trouvent un certain écho auprès des organismes officiels. En 1969, les ministères de l'Équipement et du Logement organisent un concours - le Grand Prix international d'urbanisme et d'architecture - sur le thème "Construction et Humanisme ». Treize projets sont retenus et exposés lors de la première semaine des Rencontres internationales d'urbanisme et d'architecture qui se tiennent à Cannes en mars 1969. Parmi eux figurent celui élaboré par Yona Friedman et Guy Rottier ${ }^{38}$, ainsi que ceux de Pascal et Claude Häusermann-Costy, d'André Biro et Jean-Jacques Fernier et de Jean-Louis Chanéac, architectes qui font tous partie du GIAP ${ }^{39}$. Nice Futur se situe à la croisée des réflexions de Yona Friedman sur les villes spatiales et de celles de Guy Rottier sur les architectures mobiles. Sur le littoral niçois, au niveau de l'embouchure du $\operatorname{Var}^{40}$, ils proposent d'élever une cité de 500000 habitants et une unité de tourisme permettant d'accueillir 300000 vacanciers. La cité s'articule autour de trois immeubles "serpents " de 20 kilomètres de long et 50 mètres de large, longeant le lit du Var (fig. 14). À la 
jonction de ces trois immeubles, des tours " hélices » de 85 niveaux jouent le rôle de signaux verticaux. L'unité de vacances constitue une application de l'urbanisme spatial de Yona Friedman. Elle se compose d'une ossature tridimensionnelle de 4 à 6 niveaux reposant sur des pilotis de 40 à 60 mètres de hauteur servant à la circulation verticale. Celle des piétons est assurée par des passerelles qui servent également de lieux d'échange et de loisir (commerces, promenades, embarcadères, etc.). Avec Nice Futur, Yona Friedman et Guy Rottier inventent une «structure » urbaine prolongeant les réflexions de Michel Ragon quant à l'architecture-sculpture et mêlant étroitement approches formelles et fonctionnelles.

Figure 14

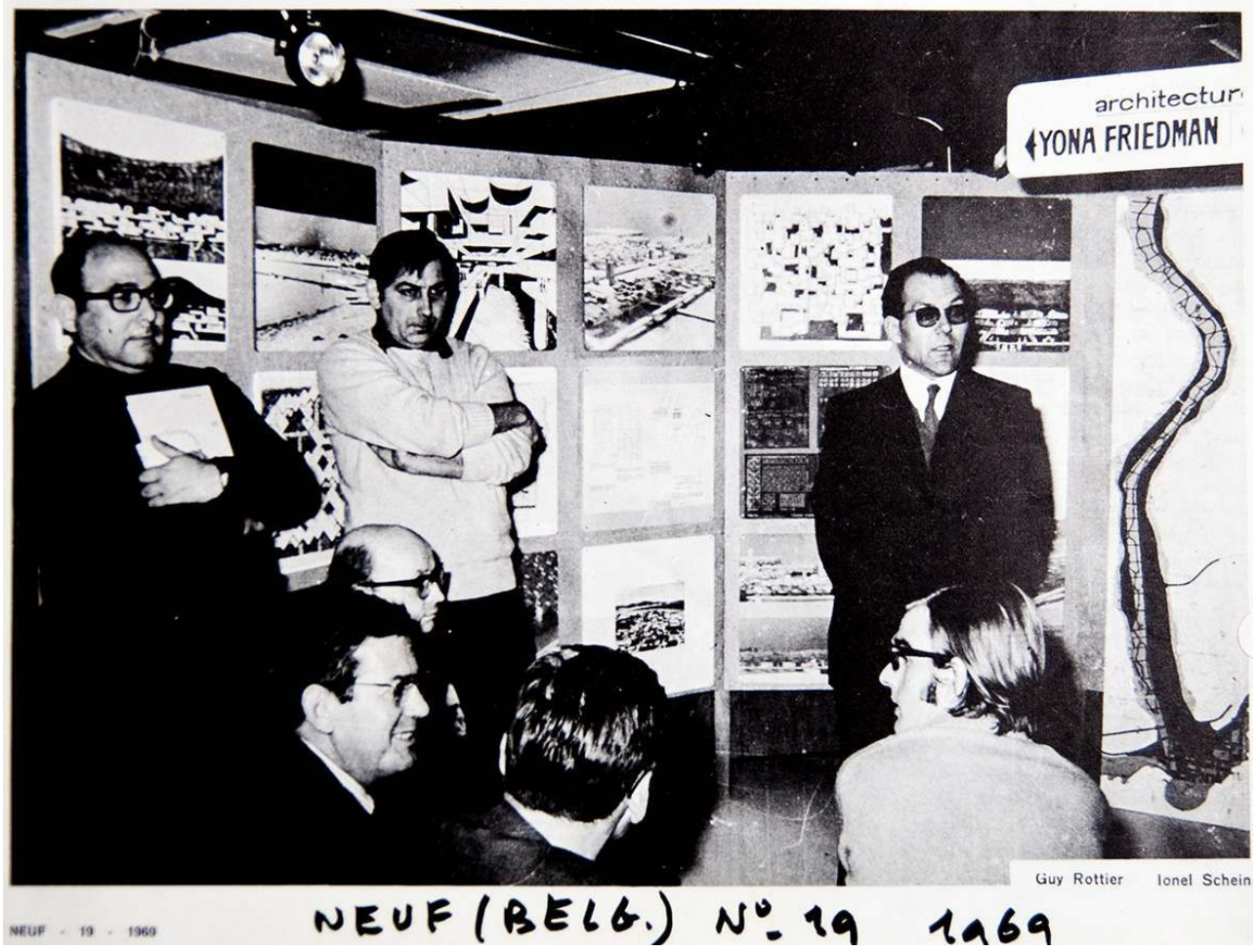

Guy Rottier présentant son projet Nice-Futur au Concours international urbanisme et architecture à Cannes en mars 1969. Document tiré de Guy Rottier, "Guy Rottier et Yona Friedman, Nice au Futur », Neuf, $n^{\circ} 29,1969$

(C) MAMAC, FONDS GUY ROTTIER, 72 M ROTT.

\section{Les « Conspiratifs », ultime tentative de Guy Rottier pour prolonger les collaborations entre architectes et artistes}

12 À partir de 1970, Guy Rottier délaisse toute pratique architecturale et se consacre à une activité purement théorique et artistique. Depuis la Syrie (1970-1978) et le Maroc (1978-1986) où il réside désormais, il élabore près de cinquante-sept projets, organise des expositions et publie des essais sur l'architecture et l'urbanisme ${ }^{41}$. À son retour à Nice en 1987, Guy Rottier crée le groupe des «Conspiratifs ». Son objectif est de faire revivre la flamme créatrice qui avait animé la Côte d'Azur durant les années 1960-1970, en 
réunissant artistes, architectes, designers, journalistes et «toutes personnes pouvant apporter quelque chose de nouveau au contexte de la commande architecturale » (fig. 15)

Figure 15

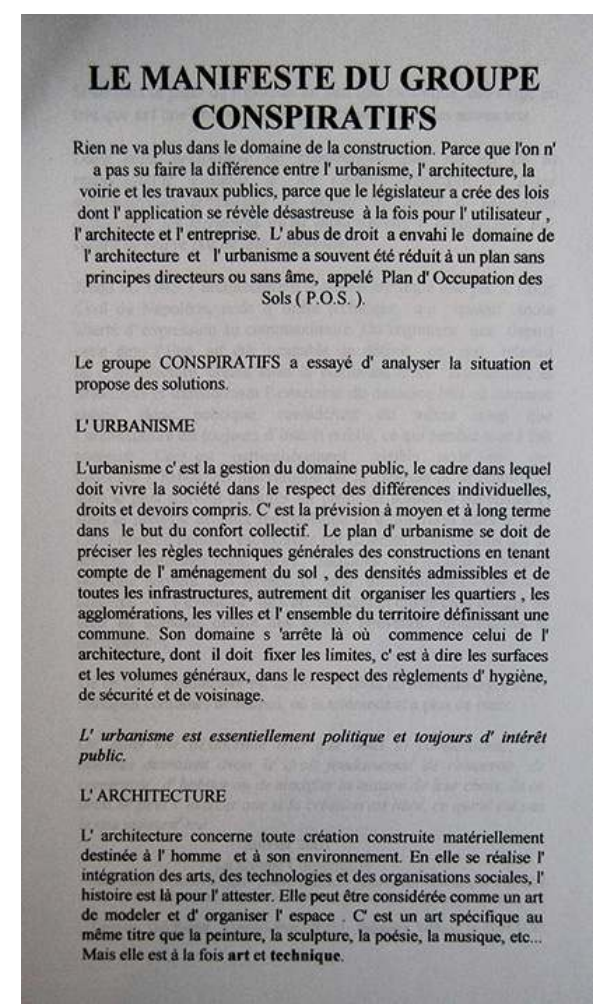

MANIFESTE DU gROUPE LES CONSPIRATIFS, 1996.

(C) ARCHIVES PRIVÉES ODETTE BARBERIS-ROTTIER, 2014.

13 La création du groupe intervient le 12 mars 1996 au cours d'une table ronde ${ }^{42}$ qui se tient au musée d'Art moderne et d'Art contemporain de Nice (MAMAC Nice), institution culturelle récemment créée voulant incarner l'importance de l'École de Nice ${ }^{43}$. Au sein du nouveau collectif, Guy Rottier entend raviver l'ancien cercle mais aussi établir des liens avec les créateurs plus jeunes. Dans un texte au titre un tant soit peu provocateur «Conspiratifs, Faut-il continuer à nourrir les maisons aux farines provençales? », Guy Rottier indique que

le groupe Conspiratifs se veut hors la loi, indépendant de tout organisme officiel ou politique et ne comporte que des membres éphémères (ceux qui sont présents aux réunions), il comporte cependant un seul membre d'honneur, Michel Ragon, en remerciement pour tout ce qu'il a fait pour l'architecture prospective dès les années $1960^{44}$.

Dans le manifeste des "Conspiratifs », Guy Rottier revendique la liberté de création en matière d'art et d'architecture, dénonce les règles qui encadrent l'aspect visuel des constructions, leurs dimensions et l'aménagement de leurs abords ${ }^{45}$. L'objectif affiché est d'aboutir à une architecture de qualité et de conserver un rapport harmonieux avec le contexte, ce qui était déjà revendiqué par Guy Rottier dans les années 1960.

La dissolution du groupe des «Conspiratifs » à la fin de l'année 2012, peu avant le décès de Guy Rottier (juin 2013), confirme que ce collectif reposait sur sa volonté individuelle. 
Néanmoins, le groupe fut assez actif. Lors d'une exposition collective (MAMAC, 1998), architectes et artistes présentent côte à côte leurs projets pour Nice à l'horizon de l'an $2000^{46}$ (fig. 16). L'exposition d'architecture, de design et de dessins humoristiques au château de La Napoule (Alpes-Maritimes, juin 1997), la création du journal Conspiratifs (1998) et la participation à la rencontre organisée par l'ordre des architectes de la région PACA (Saint-Maximin, juillet 1997) témoignent d'un certain dynamisme. Si Guy Rottier réussit indéniablement à temporairement donner un nouveau souffle à une pratique collective de l'invention, son rêve utopique d'abolir le système de la commande publique et de réunir architectes et artistes autour de visions communes est néanmoins mis en échec. Force est de constater que la notion du collectif dans les années 1990 et 2000 est très différente de celle des années 1960: la mondialisation de l'art et de l'architecture, leur « starisation » et l'hyperconsommation en ayant parfois fait des biens consommables où politique, économique et social interagissent de manière différente.

Figure 16

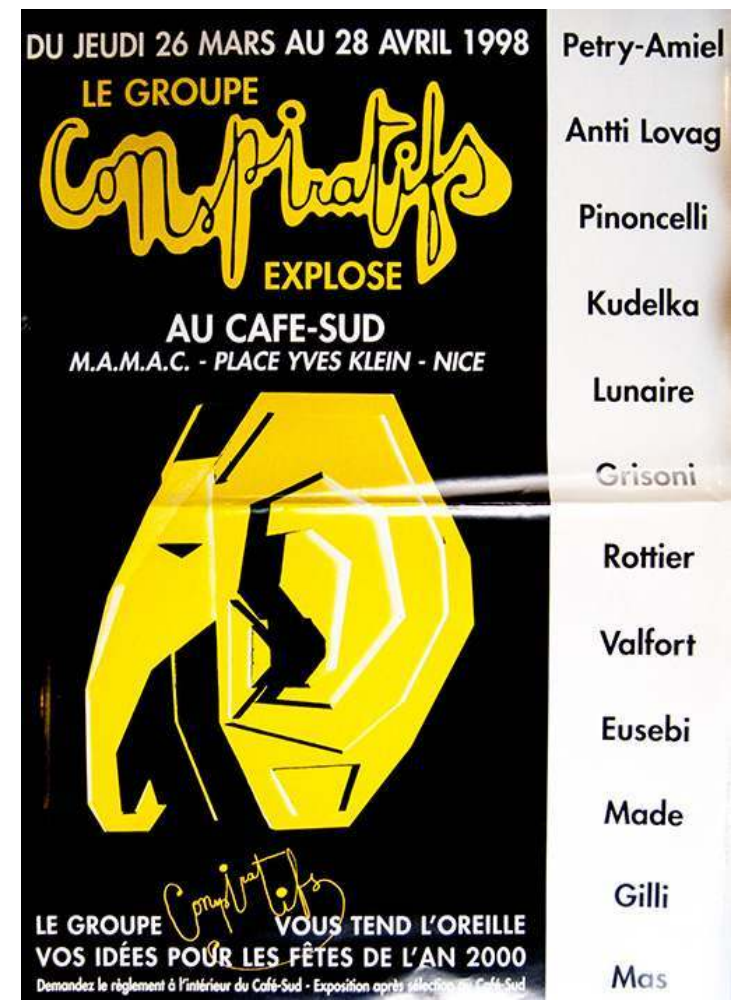

Affiche de l'exposition Idées pour l'an 2000 (MAMAC Nice, 1998).

(C) ARCHIVES PRIVÉES ODETTE BARBERIS-ROTTIER, 2014.

\section{L'hypothèse d'une architecture devenant « arTchitecture »}

En 1987, Guy Rottier avait pourtant commencé à parler d'une « arTchitecture » désignant sa propre pratique comme une fusion de l'art et de l'architecture ${ }^{47}$. Ce néologisme lui permet de donner sens à sa vision personnelle de l'interdépendance entre architecture et art. Il considère qu'il y a une « Architecture avec un grand A » (l'architecture officielle), une «architecture avec un petit a » (une architecture sans âme ne suscitant aucune 
créativité ${ }^{48}$ ) et « l'arTchitecture, avec un grand $\mathrm{T}^{49}$ ». De cette dernière, Guy Rottier dit qu' "en dehors de tout contexte", elle est "celle des marginaux, des inventeurs, nouveaux facteurs Chevaux. Elle correspond à la création, à la nouveauté, à l'art en quelque sorte, comme la sculpture ou la peinture $»^{50}$. Pourtant, la production de Guy Rottier permet de constater qu'il existe une distance entre l'architecture que l'architecte «réalise " et celle dont il «rêve". Si la première semble prolonger la tradition corbuséenne, la seconde se démarque par des formes plus audacieuses puisant tout autant dans l'architecture-sculpture, l'architecture parlante, l'art de l'assemblage et de la récupération que dans le nouveau réalisme.

$\mathrm{Au}$ début de sa vie professionnelle, Guy Rottier a en effet réalisé plusieurs maisons individuelles sur la Côte d'Azur. Ces réalisations - villas F. Rosa (Vence, 1961), villa Barberis (Villeneuve-Loubet, 1962), J. Chouvelon (Villefranche-sur-Mer, 1960), LaudeDujardin (Villefranche-sur-Mer, 1963) et Emanuel Laude (Villefranche-sur-Mer, 1964) mobilisent son savoir-faire d'ingénieur et d'architecte et s'inscrivent dans une démarche raisonnée inspirée des cinq points de l'architecture moderne définis par Le Corbusier. Audelà de cette filiation, il tente de se départir des pilotis au profit de rez-de-chaussée de forme parallélépipédique, ancrés dans le sol. Il se sert de formes géométriques élémentaires pour créer des analogies combinatoires entre les sous-espaces. Par exemple, il met l'accent sur la structure apparente de la villa Barberis et traite les éléments fonctionnels (escaliers, cheminées) de façon à en faire, à l'instar de Le Corbusier, en signifiants plastiques (fig. 17). Ainsi, par emboîtement, soustraction, extrusion, division et addition, Guy Rottier crée un jeu visuel dynamique basé sur le dualisme entre indépendance et cohérence. Il accentue l'expressivité de ses architectures par l'usage de la couleur et les textures du béton.

\section{Figure 17}

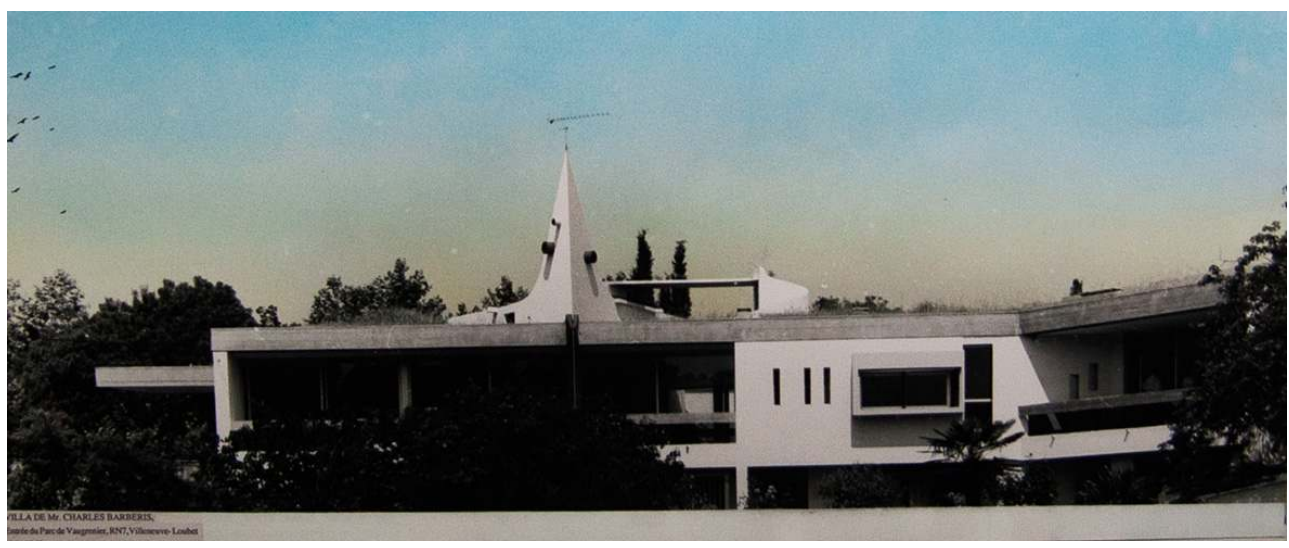

FAÇADE SUD DE LA VILLA C. BARBERIS.

(C) FONDS GUY ROTTIER, FRAC ORLÉANS, DÉPÔT GUY ROTTIER, D - 0000901.

18 Progressivement, à la faveur de sa fréquentation des artistes, cette logique formelle basée sur les combinatoires entre les formes abstraites s'estompe au profit d'une démarche plus sculpturale et plastique visant à rendre l'œuvre architecturale indépendante et parlante. Pour se libérer du fonctionnalisme, Guy Rottier s'essaie à de nouvelles expériences plastiques qui vont de l'accumulation et la récupération d'objets à des œuvres de Land Art totalement inédites, en passant par des dispositifs mobiles. L'adhésion de Guy Rottier au GIAP le confronte in situ à la notion d'architecture-sculpture fortement plébiscitée par 
Michel Ragon et le conduit, dans sa pratique personnelle, à rapprocher art et architecture. La villa du sculpteur Farhi (projet théorique, 1967) et la Maison à Gassin (projet théorique, 1969), bien que non réalisées, annoncent un nouveau formalisme où la volumétrie se complexifie (fig. 18). Par un délicat ajourage des surfaces, par l'extrusion et le mouvement rotatif des éléments constitutifs des volumes, la maison devient une structure aérienne habitable. Les lignes obliques, les formes triangulaires, le cube tronqué, les cylindres renversés annoncent une nouvelle conception de l'habitat. Ce dialogue entre formes et spatialité participe à la création d'une esthétique propre à Guy Rottier dans laquelle, comme dans une performance artistique, le mouvement établit de nouvelles connexions entre espaces habitables et paysage. Les capsules mobiles triangulaires accrochées aux câbles de la toiture végétalisée de la villa Arman (1968) illustrent cette idée d'une architecture destinée à être déplacée, démontée et remplacée. L'architecture ne se limite plus à des éléments statiques, elle est désormais définie dans l'espace-temps.

Figure 18

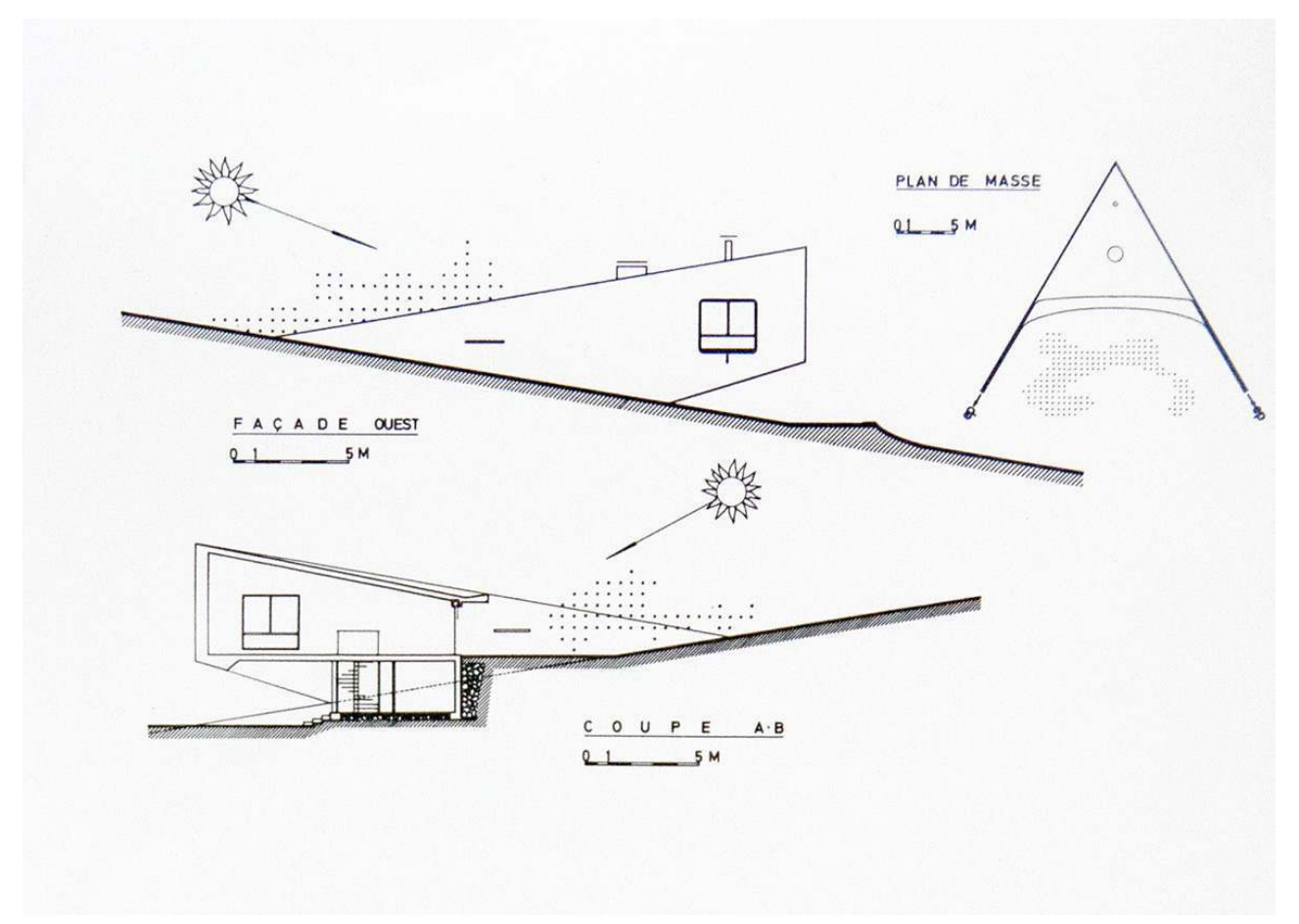

PLAN MASSE, FAÇAde OUESt ET COUPE A-B (EST-OUEST) DE LA MAISON À GASSIN (1968-1969, NON RÉALISÉE).

(C) ARCHIVES PRIVÉES D'ODETTE BARBERIS-ROTTIER, 2014.

Guy Rottier explore aussi la notion "d'architecture parlante » au travers de formes architecturales figuratives. Il emprunte cette notion au XVIII ${ }^{\mathrm{e}}$ siècle $^{51}$, et notamment à Claude-Nicolas Ledoux mais aussi au Nouveau Réalisme et au concept d'architecture « canard » de Robert Venturi et Denise Scott Brown ${ }^{52}$. Guy Rottier considère en effet que les formes parlantes dépassent la simple imitation de la fonction architecturale pour contribuer à une lecture sémiotique et poiétique du projet. La série de maisons industrialisées enterrées qu'il imagine à partir de 1964 démontre qu'il considère que la maison dépasse son usage fonctionnel pour se charger de messages politiques et publicitaires et, s'inspirant du Land Art, Guy Rottier manipule le végétal pour dessiner 
des slogans sur la toiture. Les textures et les couleurs qu'il utilise sont celles du paysage. Dans le même état d'esprit, il imagine des Maisons cibles (projets théoriques, 1978) pour dénoncer les régimes politiques autoritaires de certains pays ${ }^{53}$. L'architecture devient capable de renseigner sur le monde qui l'entoure (fig. 19).

\section{Figure 19}

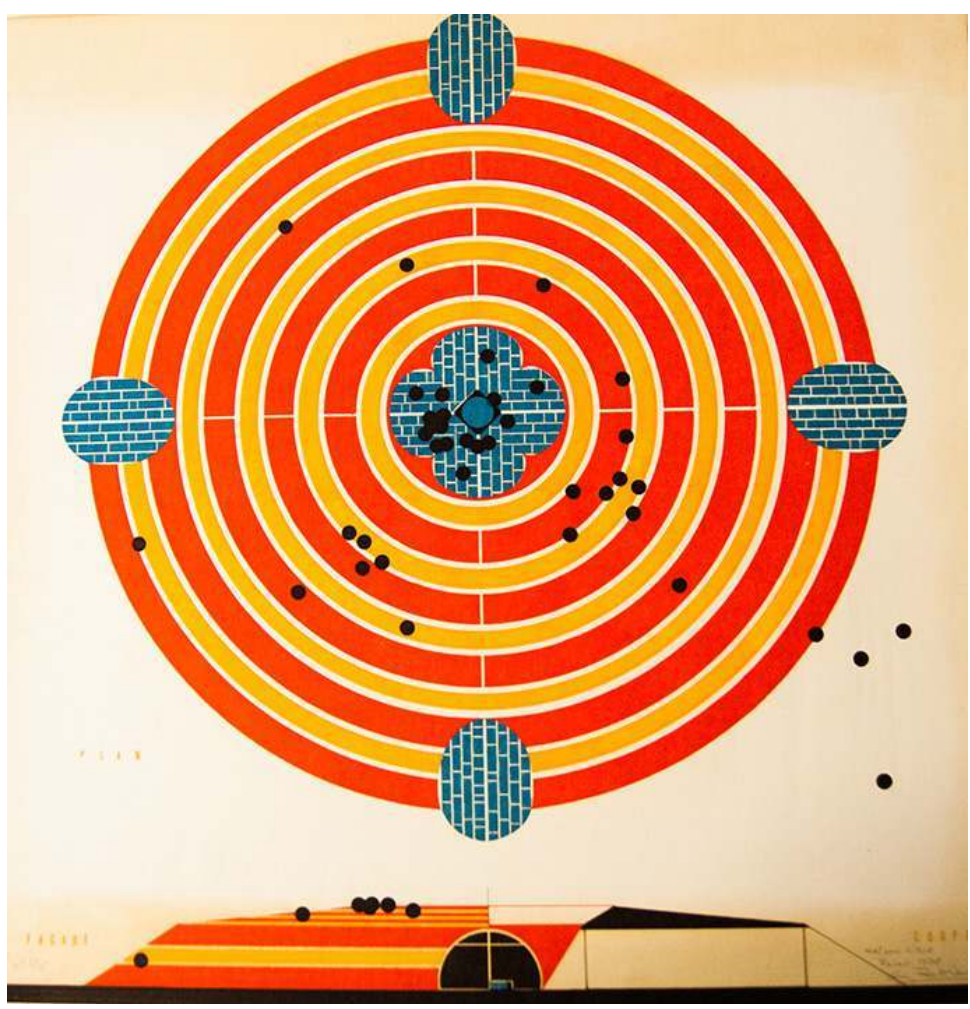

PLAN ET FAÇADE de LA MAISON ENTERRÉE « CIBLE » (1978), gOUACHE ET ENCRE SUR PAPIER, 50 x 50 CM.

(C) FRAC ORLÉANS, FONDS GUY ROTTIER, NON COTÉ.

Parfois l'architecte quitte le registre politique pour se cantonner au mimétisme organique. La Maison escargot (projet théorique, 1964) met en scène les rapports complexes et mathématiques qui régissent les formes vivantes. Ce projet, qui a influencé l'œuvre de Claude Gilli, tend à plaider pour une relation complète et harmonieuse des formes architecturales. En adoptant des formes de serpent, de main, de soleil ou de visage, les maisons de Guy Rottier projettent l'architecture dans des univers énigmatiques où la variété et l'abondance des matériaux multiplient les possibilités plastiques fournies par l'environnement immédiat. Si Arman utilise tout type d'objet pour réaliser ses œuvres, si César fait du pouce un symbole du Réalisme et si Ben fait du graphisme un outil d'expression, Guy Rottier s'emploie à mettre en évidence le fait que l'architecture ne se réduit pas à une enveloppe fonctionnelle. En 1984, il imagine par exemple une façade en flammes pour une compagnie d'assurances, un bâtiment en forme de radio pour le siège social d'une radio à Rabat, une cabine téléphonique en forme d'oreille, un propulseur pour un château d'eau à Damas (fig. 20). Il tente ainsi de donner sens à des architectures objet. Ces dernières, extraverties et signifiantes, établissent un dialogue direct avec l'usager. Par ailleurs, Guy Rottier s'inspire, dès 1965, de Kurt Schwitters pour créer des architectures obtenues par des processus d'accumulation, d'assemblage, de collage et 
d'addition. Un élément simple comme l'autobus peut désormais se transformer, par accumulation, en tours verticales, en hôtel ou en école (fig. 21). Pour Guy Rottier, l'objet trouvé est susceptible de se transformer en architecture-sculpture. Il n'en demeure pas moins qu'elle intègre un rapport harmonieux entre forme et fonction.

Figure 20

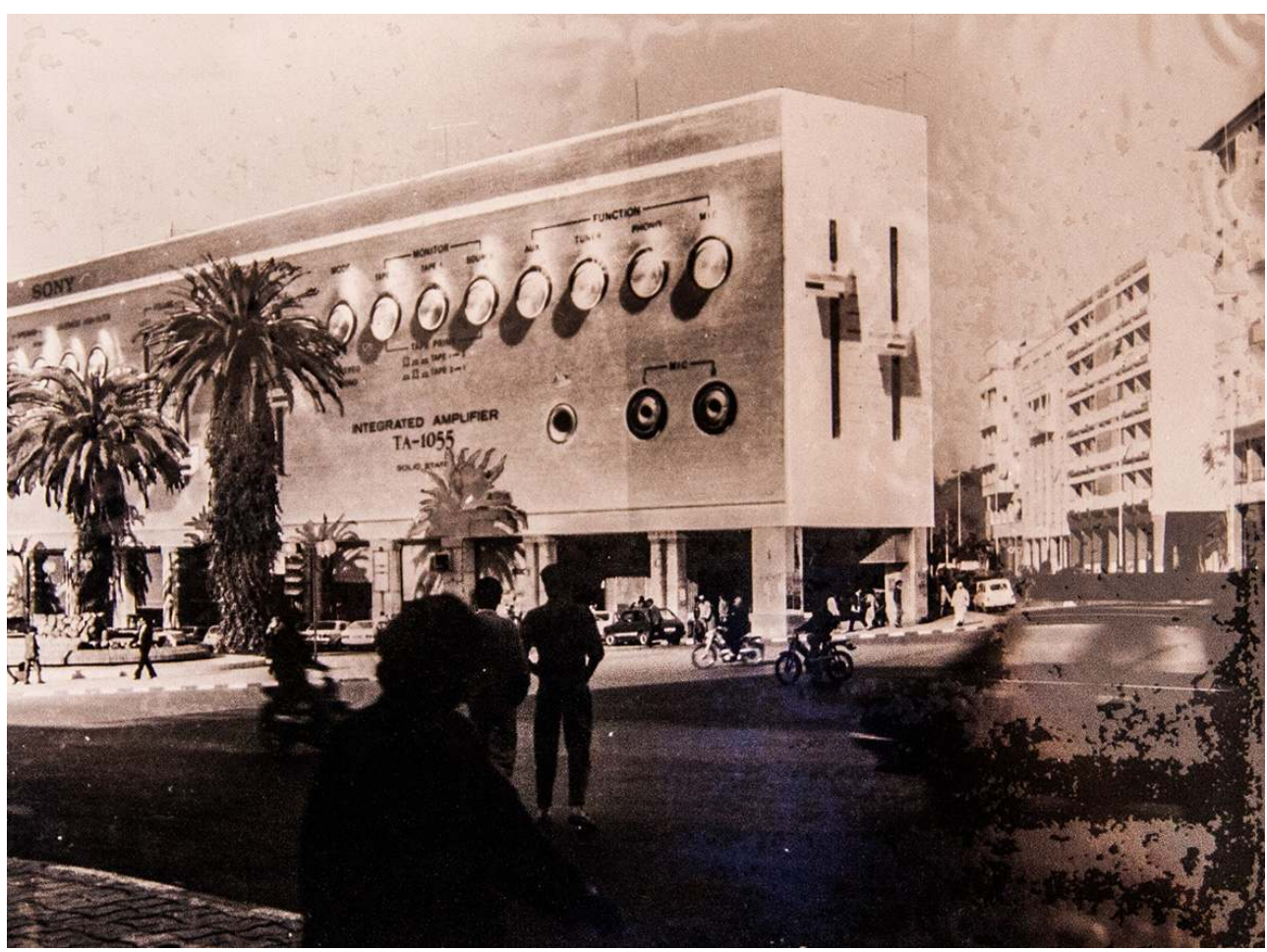

PHOTOMONTAgE de L'IMMEUbLe RADIO À RABAT (MAROC, 1986), 50 X 60 CM.

(C) ARCHIVES PRIVÉES D'ODETTE BARBERIS-ROTTIER, 2014. 


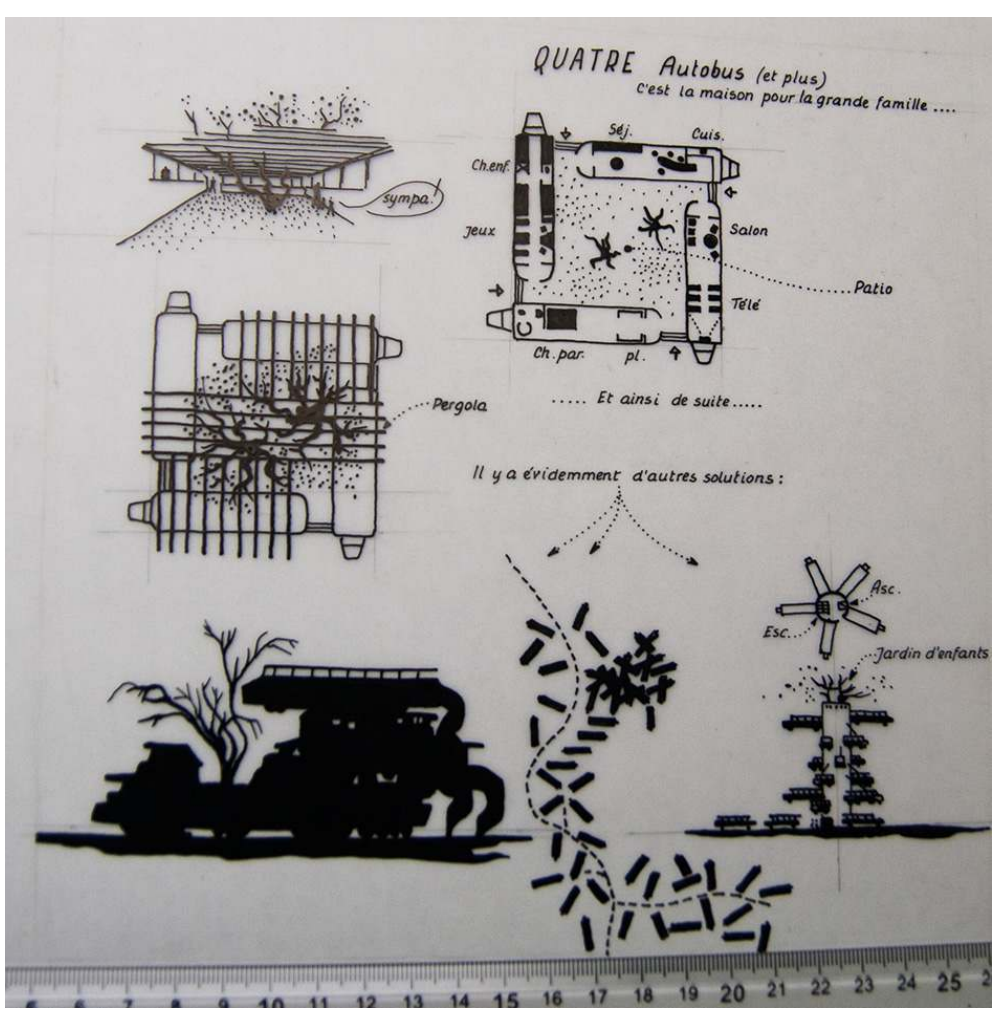

VILLAgE DE VACANCES, PRINCIPE DE L'ARCHITECTURE D'OCCASION (ÉTUDE THÉORIQUE, 1967), ENCRE de ChINe SUR CALQUe, 50 × $65 \mathrm{~cm}$.

(C) FRAC ORLÉANS, FONDS GUY ROTTIER, NON COTÉ.

21 En s'inspirant des formes élémentaires, archétypales et organiques, Guy Rottier est à la fois artiste, ingénieur et architecte. Il crée une architectonique singulière nourrie par une approche plastique complexe et hétérogène s'inspirant du fonctionnalisme et $d u$ brutalisme de Le Corbusier, de l'audace des membres du GIAP, de l'humour acide de JeanMarc Reiser et de la liberté créatrice des artistes de l'École de Nice. En empruntant à l'œuvre d'art sa poïétique, la limite entre art et architecture est ainsi gommée et Guy Rottier crée son propre langage plastique : «l'arTchitecture ». Pour pousser plus loin la transdisciplinarité, Guy Rottier emprunte à l'art ses démarches conceptuelles. Du happening à la récupération, en passant par la peinture, le design, les sérigraphies, il n'hésite pas à multiplier les expériences créatives. Au cours de sa carrière, il organise cent cinq expositions où seront présentés côte à côte les projets les plus insolites. Toutefois, malgré cette porosité artistique, l'architecture reste la ligne directrice de Guy Rottier et toutes ses réflexions portent sur la ville du futur et le contexte urbain au travers de la génération d'espaces mobiles et éphémères. Se servant de tout type de medium, il dépasse la pratique traditionnelle de l'architecture pour intégrer d'autres modalités de création : dessins humoristiques, livres d'artistes. Il classe ses peintures par séries et réalise des sculptures qu'il réutilise dans l'espace architectural comme sa série d'échiquiers (1979) qui servent de maquette pour un projet de Maison enterrée échiquier (projet théorique), 1978 (fig. 22). 
Figure 22

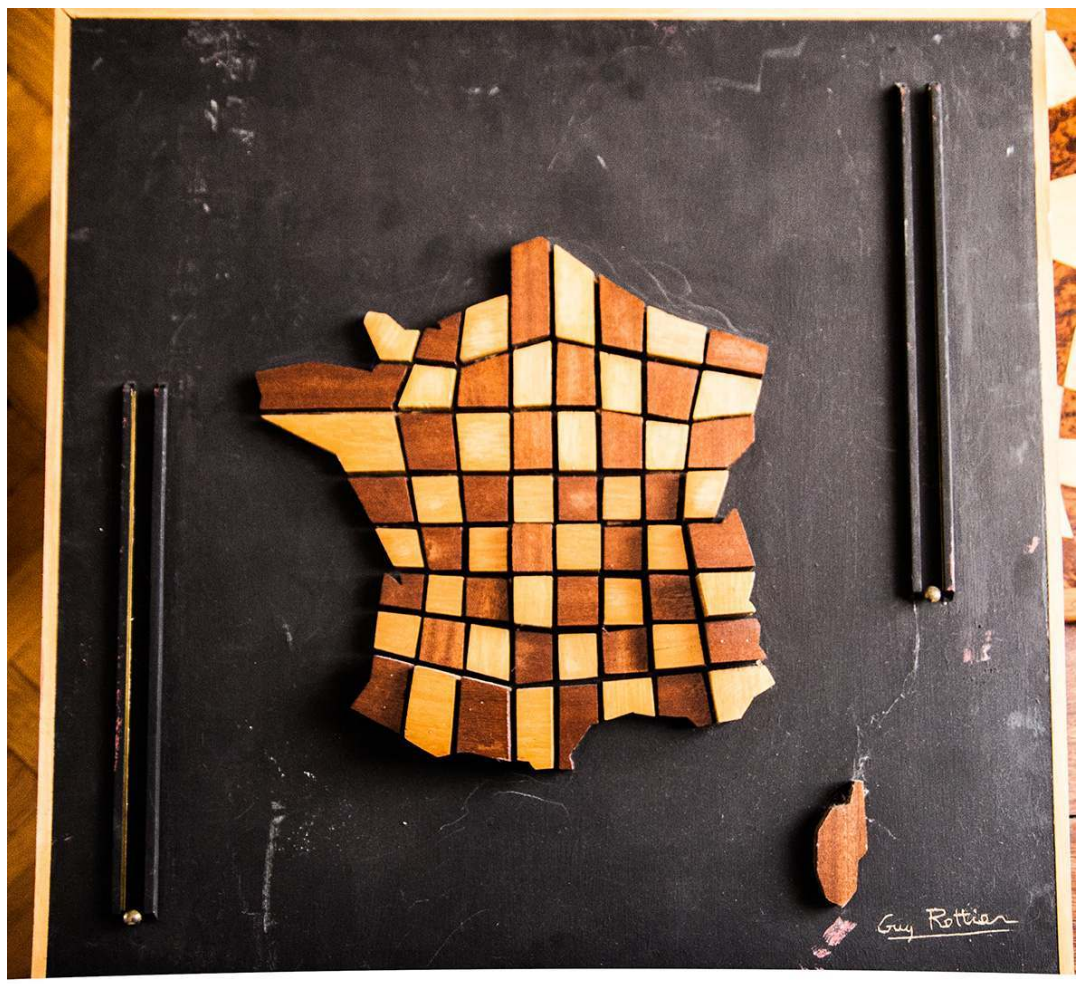

ÉCHIQUier EN RELIEF, CARTE de FRANCE (1985), hÊTRE ÉTUVÉ ET WENgÉ.

(c) ARCHIVES PRIVÉES D'ODETTE BARBERIS-ROTTIER.

\section{Le collectif, un incubateur pour l'architecte?}

Au regard du parcours de Guy Rottier, il est évident que le collectif a agi sur lui comme un incubateur. Partager, discuter, évaluer et échanger avec les artistes et intellectuels lui ont permis de se positionner dans une perspective de prospective architecturale. Cela peut être interprété comme un prolongement naturel de son apprentissage au sein de l'agence de Le Corbusier mais aussi, plus prosaïquement, comme une opportunité pour Guy Rottier de promouvoir ses idées. Son expérience atypique permet de mettre en lumière l'effervescence créatrice des années 1960 et son aspiration à dépasser, à transcender les disciplines par le biais du collectif, mais elle révèle aussi et surtout les inévitables frictions entre l'individu et le groupe. Guy Rottier a peut-être, en un sens, expérimenté la synthèse des arts réclamée par Le Corbusier. Mais cette expérience collective n'a pas été sans difficultés et elle a abouti à de nouveaux paradigmes tant en art qu'en architecture. En cela, le qualificatif d' " arTchitecte » désigne assez justement la pratique polymorphe de peintre, de photographe, d'illustrateur, de designer, de musicien, d'ingénieur et d'architecte de Guy Rottier. 


\section{NOTES}

1. - ECHEIKH-EZAOUALI BABAY, Nouha. Guy Rottier (1922-2013). Réflexions prospectives et utopies architecturales, mémoire de master 2 recherche en histoire de l'architecture sous la direction d'Éléonore Marantz, université Paris-1-Panthéon-Sorbonne, 2015. Toutes les informations biographiques relatives à Guy Rottier contenues dans cet article ont été établies dans le cadre de ce travail de recherche, le premier à être consacré à l'architecte.

2. - La Middelbare Technische School (MTS) est aujourd'hui la Hogere Technische School (HTS) ; voir l'article sur les origines de l'École technique supérieure (HTS) sur le site: http:// www.isgeschiedenis.nl/citaat-uit-het-nieuws/ontstaan-van-de-hogere-technischeschool-hts/ [consulté le 07/01/2015].

3. - « In the years of the Nazi dictatorship, the company's scientific and production potential was increasingly focused on equipping the German armed forces. Airplanes, submarines, tanks and guns were fitted with Zeiss instruments. After the unconditional surrender of the German armed forces, the allies also held Zeiss responsible for its contribution to the preparation and conducting of the Nazi war of aggression. " - The Carl Zeiss Foundation in Jena - Site Officiel du groupe Zeiss : https://www.zeiss.com/corporate/int/history/company-history/at-aglance.html\#inpagetabs-1 [consulté le 23/07/2017].

4. - ROTTIER, Guy. Guy Rottier un arTchitecte, mémoires dactylographiées rédigées par Guy Rottier, 2003, p. 4 (déposées aux AD Alpes-Maritimes, 206 J 02).

5. - LEMOINE, Bertrand. La Cité internationale universitaire de Paris. Paris : Hervas, 1990.

6. - Dossier d'élève à l'École des beaux-arts de Paris, $13^{\mathrm{e}}$ promotion (AN, AJ/52/1328).

7. - AMOUROUX, Dominique. André Wogensky. Paris : Éditions du Patrimoine, collection «Carnets d'architectes, $\mathrm{n}^{\circ} 11 », 2011$.

8. - SBRIGLIO, Jacques. L'Unité d'habitation de Marseille. Le Corbusier. Marseille : Parenthèses, 2013, p. 116 ; liste des personnalités ayant travaillé pour l'Atelier des bâtisseurs (ATBAT).

9. - Charles Barberis, grand ami de Le Corbusier, est un menuisier et entrepreneur installé à Villeneuve-Loubet (Alpes-Maritimes) qui avait fourni les menuiseries de l'Unité d'habitation de Marseille et du cabanon de Roquebrune-Cap-Martin (en 1949). Il réalise aussi les deux prototypes en bois des Modulor $\mathrm{n}^{\circ} 1$ et $\mathrm{n}^{\circ} 2$ de Le Corbusier. Guy Rottier le croise sur le chantier de l'Unité d'habitation de Marseille en 1948. Dix ans après, Charles Barberis s'associe à Guy Rottier ; c'est le début de longues années d'aventures placées sous le signe de la réussite et de l'échec. C'est grâce à cette rencontre que sont nés les cabanons de vacances U.V.366, le Cabanon pour enfants C.C. 215 et la Maison volante (1964).

10. - Les articles sur la Maison volante sont conservés au sein du fonds Guy Rottier au MAMAC de Nice sous forme de coupures de presse : «Casa volante». Domus, $\mathrm{n}^{\circ} 425,1965$; « For cable cars? For helicopters?». Progressive architecture, 1965; «Week-end cottage becomes airborne». Architect and builder, 1964 ; « La maison volante ». Bauwelt, $\mathrm{n}^{\circ} 33$, août 1964.

11. - Guy Rottier rend compte de sa rencontre avec Bernar Venet: «En 1965, j'ai reçu à mon bureau de Nice un jeune artiste, Bernar Venet, qui avait vu mes publications dans certaines revues. Enthousiasmé par mes premières recherches, il voulait en savoir plus. Tout d'abord, il m'a présenté quelques amis et j'ai découvert à travers lui une petite fourmilière artistique très active qui travaillait à Nice, dans laquelle je me suis incorporé naturellement et dont les artistes sont devenus très rapidement mes amis. » (ROTTIER, Guy. Mémoires cit., p. 21).

12. - GOLDBERG, Roselee, PEDRINI, Enrico, SAUSSET, Damien. Bernar Venet: performances, etc. 1961-2006. Milan/New York: Charta, 2007, p. 28. 
13. - Liste des projets théoriques : Maison enterrée à carcasses de voitures, pneus, gazon pour Ben (1972), Maison enterrée terrasse avec végétation (1972), Maison enterrée terrasse en eau (1972), Maison à caractère publicitaire « La vache qui rit » (1972), Maison à caractère publicitaire « Top Model » (1972), Maison enterrée, jardin en forme de jeu d'échec (1978), Maisons enterrées «Cibles » (1978), Maison enterrée Serpent (1979), Maison enterrée Soleil (1979).

14. - Plus communément connue aujourd'hui sous le nom de villa Arson.

15. - LECLERC, Franck, HUTCHINGS, Ralph. L'école de Nice: paroles d'artistes. Paris : Verlhac, 2010, p. 27.

16. - Ibid., p. 17.

17. - O'NEILL, Rosemary. Art and Visual Culture on the French Riviera, 1956-1971: The École de Nice. Farnham/Burlington: Ashgate, 2012, p. 4. Rosemary O'Neill note que "profitant de leur proximité spatiale et d'un environnement artistique ouvert, véritable laboratoire d'expérimentations, les artistes niçois ont bénéficié de liens établis avec des groupes bien définis que l'on identifie comme parties prenantes de l'École de Nice - ceux du Nouveau Réalisme, d'Art Total et Fluxus, de Supports-Surfaces, du groupe 7 - mais aussi d'alliance et d'interactions souples, qui avaient tendance à estomper les frontières artistiques, permettent ainsi une interaction fertile entre ces différents artistes ».

18. - SEMIN, Didier. "Fluxus". Dans Encyclopcedia Universalis, voir le site: http:// www.universalis-edu.com/encyclopedie/fluxus/ [consulté le 06/01/2014].

19. - BIANCHERI, Alain, GIORDAN, André, FRANÇOIS, Rébecca. L'École de Nice. Nice : Ovadia, 2007, p. 36 .

20. - RIVIÈRE, Claude. « La charge solaire de l'artiste ». Combat, 22 août 1960.

21. - VALDMAN, Édouard. Le Roman de l'École de Nice. Paris : La Différence, 1991, p. 31.

22. - Les principales expositions auxquelles participe Guy Rottier aux côtés des artistes de l'École de Nice sont : « Un architecte - un peintre - un photographe », avec le peintre Bernar Venet et le photographe Alain Sèbe (1967) ; «École de Nice » à la galerie Alexandre de La Salle (1997) ; «Guy Rottier, architecte de l'École de Nice » au MAMAC Nice (1998) ; «Le Paradoxe d'Alexandre (de la Salle), un parcours : 1960-2000 " au Centre international d'art contemporain, château de Carros (2000).

23. - ROTTIER, Guy. Mémoires cit., p. 22.

24. - JORNOD, Jean-Pierre, JORNOD, Naïma, MENZ, Cäsar. Le Corbusier ou la synthèse des arts. Cat. exp., Genève, musée Rath, 9 mars-6 août 2006. Genève/Milan : musées d'Art et d'Histoire/Skira, 2006.

25. - ROTTIER, Guy. ArTchitecte de l'insolite : Unusual Architecture. Nice : Z'éditions, 1990, p. 44.

26. - BOUCHIER, Martine. «L'art n'est pas l'architecture ». Dans YOUNÈS, Chris (dir.). Art et philosophie, ville et architecture. Paris : La Découverte, coll. « Armillaire », 2003, p. 110.

27. - Parmi les membres fondateurs figurent Michel Ragon, Yona Friedman, Paul Maymont, Ionel Schein, Nicolas Schöffer, Walter Joans et Georges Patrix.

28. - LEEMAN, Richard, JANNIÈRE, Hélène (dir.). Michel Ragon critique d'art et d'architecture. Rennes : PUR, 2013.

29. - JANNIÈRE, Hélène. «L'urbanisme contre la ville : Michel Ragon et la critique de la ville années 1960-1970 ». Dans Ibid., p. 183.

30. - RAGON, Michel. Le livre de l'architecture moderne. Paris : R. Laffont, 1958.

31. - ROTTIER, Guy. Mémoires cit. (AD Alpes-Maritimes, 206 J 02).

32. - « Maison escargot », « Village sur fil », « Ferienstadt am Seilnetz », « Ein wachsendes Haus ». Bauwelt, $\mathrm{n}^{\circ} 48$, novembre 1968 .

33. - Michel Ragon décrit la ville spatiale de Yona Friedman comme « une structure d'acier tridimensionnelle, placée sur de hauts pilotis, et formant un maillage dans lequel peuvent être insérés tous les éléments urbains. " (RAGON, Michel. L'homme et les villes [1975]. Paris : Albin Michel, 1995, p. 208). 
34. - La ville flottante de Paul Maymont est composée « de caissons flottants de forme conique. Il s'agit en fait de cités-champignons reliés au sol [...] par une colonne centrale creuse, en béton, de vingt mètres de diamètre, représentant à la fois la circulation verticale et le grand collecteur. » (RAGON, M. Où vivrons-nous demain ? Paris : Robert Laffont, 1963, p. 136).

35. - Le « terme d'architecture-sculpture, [...] apparaît pour la première fois dans [...] Où vivronsnous demain ? édité à Paris en 1963 [...]. Le terme est apparu dans ses deux variantes : sculpturearchitecture et architecture-sculpture désignant chez Ragon le travail des architectes novateurs développant les effets de plasticité dans l'architecture, aussi bien que celui des artistes de l'aprèsguerre, dont le travail était une tentative de transgression du genre » (CYTLAK, Katarzyna Maria. Les utopies grises : projets architecturaux d'Alex Mlynárčik, Tadeusz Kantor et Jozef Jankovič dans l'Europe centrale des années soixante-dix. Thèse de doctorat en histoire de l'art dirigée par Philippe Dagen. Paris : université Panthéon-Sorbonne, 2012, p. 97-99.

36. - RAGON, Michel. Où vivrons-nous demain ? Op. cit.

37. - JANNIÈRE, Hélène. Op. cit., p. 193. Michel Ragon considère que «les deux principales expositions du GIAP ont été [...] les douze villes prospectives présentées pour la première fois, en juillet 1965, au siège de la Compagnie de Saint-Gobain à Neuilly [...], puis en octobrenovembre 1966, l'exposition de tous les membres du GIAP "Architecture prospective » à la galerie Arnaud à Paris» (RAGON, Michel. «Le groupe international d'architecture prospective, deux ans d'activité ». Cimaise, $n^{\circ} 79,1967$, p. 42-51).

38. - «Le Grand Prix International d'Urbanisme et d'Architecture : M. Guy Rottier, architecte niçois ». Nice matin, 31 janvier 1969 (coupure de presse, fonds Guy Rottier, MAMAC Nice, non coté).

39. - «Treize projets retenus par le jury international pour la $1^{\text {re }}$ semaine d'urbanisme et d'architecture de Cannes ». Nice-Matin, 29 janvier 1969 (coupure de presse, fonds Guy Rottier, MAMAC Nice, non coté).

40. - Ce site est actuellement occupé par l'aéroport Nice-Côte d'Azur.

41. - ROTTIER, Guy. Architecture libre. Paris : Éditions Alternatives, 1986, non paginé [ca 144 p.].

42. - La table ronde a pour thème "Architecture, design, création et tradition ». Ses membres fondateurs sont: Antti Lovag (architecte), Pierre Roche (écrivain), Thierry Valfort (architecte), Frédéric Altmann (critique d'art), Robert Kudelka (journaliste) et Dominique Pétry-Amiel (architecte). Ce noyau initial est ensuite rejoint par plusieurs membres actifs parmi lesquels Claude Gilli (artiste de l'École de Nice), Jean Mass (artiste de l'École de Nice), Yves Bayard (architecte), Yves Rottier (artiste et fils de Guy Rottier).

43. - Le MAMAC: «Le musée d'Art moderne et d'Art contemporain de Nice a été inauguré le 21 juin 1990. Plusieurs projets cohérents pour la création d'un tel musée apparaissent à Nice dans la seconde moitié de ce siècle. En 1985, l'exposition "Autour de Nice" à Acropolis présentant un premier rassemblement d'œuvres des Nouveaux Réalistes, de l'École de Nice et de Supports/ Surfaces notamment, va accréditer la nécessaire implantation d'un musée d'art moderne et d'art contemporain à Nice.» Voir le site: http://www.mamac-nice.org/francais/ [consulté le 21/07/2016].

44. - ROTTIER, Guy. Conspiratifs, Faut-il continuer à nourrir les maisons aux farines provençales? (AD Alpes-Maritimes, $206 \mathrm{~J} \mathrm{02,} \mathrm{2001,} \mathrm{p.} \mathrm{2).}$

45. - «Rien ne va plus dans le domaine de la construction, parce que l'on n'a pas su faire la différence entre l'urbanisme, l'architecture, la voirie et les travaux publics, parce que le législateur a créé des lois dont l'application se révèle désastreuse à la fois pour l'utilisateur, l'architecte et l'entreprise. L'abus de droit a envahi le domaine de l'architecture et l'urbanisme a souvent été réduit à un plan sans principes directeurs ou sans âme appelé plan d'occupation des sols (POS) », extrait du Manifeste du groupe des « Conspiratifs» (Ibid., p. 26).

46. - Cette exposition a eu une retombée à l'échelle régionale et a fait l'objet de commentaires élogieux dans la presse locale, sans toutefois dépasser le cadre de la région. 
47. - En se référant à un article rédigé par Guy Rottier et publié en 1987 dans Edino Magazine $\left(\mathrm{n}^{\circ} 1\right)$ sous l'intitulé «ART...Chitecture », on suppose que le concept d'«arTchitecture " a été évoqué pour la première fois à ce moment-là. Par la suite, c'est la publication de l'ouvrage de Guy Rottier ArTchitecte de l'insolite : Unusual Architecture, en 1991, qui va diffuser la notion d'arTchitecture auprès de la presse quotidienne et professionnelle. Les articles citant l'arTchitecture sont conservés sous forme de coupures de presse au MAMAC Nice dans le fonds Guy Rottier (72 M ROTT).

48. - Ibid.

49. - ROTTIER, Guy. ArTchitecte de l'insolite... Op. cit., p. 135.

50. - Ibid.

51. - MOLOK, Nicolas. "L'architecture parlante", ou Ledoux vu par les romantiques". Romantisme, $\mathrm{n}^{\circ}$ 92, 1996, p. 43-53. Voir le site: http://www.persee.fr/web/revues/home/ prescript/article/roman_0048-8593_1996_num_26_92_4264 [consulté le 10/01/2016].

52. - VENTURI, Robert, BROWN SCOTT, Denise, IZENOUR, Steven. L'enseignement de Las Vegas. Wavre: Mardaga, 2008 (nouvelle éd. de Learning from Las Vegas: the forgotten symbolism of architectural form paru en 1972 à Cambridge (Mass.) aux presses du MIT), p. 97.

53. - Guy Rottier a vécu vingt ans entre la Syrie et le Maroc, il a eu l'occasion de visiter l'Égypte, le Liban et l'Irak. Très proche de la réalité de ces pays, il n'a pas hésité à dénoncer les régimes politiques autoritaires à travers ses projets de « Maisons cibles pour chefs d'État ».

\section{RÉSUMÉS}

L'architecte franco-néerlandais Guy Rottier (1922-2013) a placé la rencontre et la collaboration entre architectes et artistes au cœur de sa démarche. Disciple de Le Corbusier, diplômé de l'École des beaux-arts en 1952, il n'a eu de cesse de dépasser les frontières entre art et architecture. Seul architecte du groupe des artistes de l'École de Nice, membre du Groupe international d'architecture prospective (GIAP) et fondateur du groupe des "Conspiratifs", ses recherches témoignent des collaborations fructueuses qui peuvent naître entre architectes et artistes. Audelà du fait qu'elle s'inscrit souvent dans une dynamique collaborative, la pratique personnelle de l'architecte possède une dimension transdisciplinaire intrinsèque qui le fait passer d'une activité purement architecturale à une pratique plastique de l'architecture. L'objectif de cet article est de revenir sur cette expérience pour comprendre ce basculement dans la démarche de Guy Rottier et pour questionner les modalités de collaboration entre artistes et architectes. L'étude de quelques projets, certains restés théoriques et d'autres ayant donné lieu à des réalisations, permet de poser quelques jalons quant au cheminement de Guy Rottier vers l'« arTchitecture », concept qu'il formule dès 1987. L'enjeu est de comprendre dans quelle mesure l'architecture devient pour lui synonyme d'une expérience globale. Enfin, à travers son expérience au sein du GIAP et des «Conspiratifs », on mesurera l'importance du collectif comme moteur de création individuelle.

The French-Dutch architect Guy Rottier (1922-2013) placed the collaboration between architects and artists at the centre of his work. He was a disciple of Le Corbusier, graduating from the Paris Ecole des Beaux-Arts in 1952. He continually blurred the boundaries between art and architecture. As the only architect in the l'École de Nice group, as a member of the Groupe international d'architecture prospective (GIAP) and as a founder of the 'Conspiratifs' group, his 
researches reflect the rewarding collaborations that can emerge between architects and artists. The architect's personal practice has an intrinsically interdisciplinary dimension going beyond its inscription in collaborative dynamics. In 1964, Guy Rottier joined the Ecole de Nice artists' group. This encounter marked a significant break in his career moving from architecture to fine arts. The ambition of this article is to revisit this experience to understand how Guy Rottier's approach suddenly changed and to raise further questions about the forms of collaboration between architects and artists. Through the study of a selection of projects, some of them theoretical, others actually carried out, the article looks at some of the stages of Rottier's progress towards 'arTchitecture', a concept he formulated in 1987. We will try to understand how far architecture became synonymous with a total work of art. To conclude, through his experience within the GIAP and Conspiratifs, we will try to understand how collective dynamics were a driving force in Rottier's individual creation

\section{INDEX}

Keywords : Guy Rottier, Willem Frederik Henri Rottier, Le Corbusier, André Wogensky, Roger Aujame, Georges Candilis, Shadrach Woods, Pirkko Kaarina Hirvelä, Márta Pán, Pierre Rosenberg, Nikos Chátzidakis, Fernand Gardien, Roggio Andreini, Charles Barberis, Bernar Venet, Arman, Ben [Vautier], Yves Klein, Martial Raysse, Michel Ragon, architect, artist, arTchitecture, collaboration, collective, École de Nice, GIAP, Conspiratifs, art, architecture, utopia, prospective Mots-clés : Guy Rottier, Willem Frederik Henri Rottier, Le Corbusier, André Wogensky, Roger Aujame, Georges Candilis, Shadrach Woods, Pirkko Kaarina Hirvelä, Márta Pán, Pierre Rosenberg, Nikos Chatzidákis, Fernand Gardien, Roggio Andreini, Charles Barberis, Bernar Venet, [Fernandez] Arman, Claude Gilli, Claude Farhi, Marcel Alloco, Ben [Benjamin Vautier], Albert Chubac, Noël Dolla, Claude Viallat, Yves Klein, Martial Raysse, Michel Ragon, architecte, artiste, arTchitecture, collaboration, collectif, École de Nice, GIAP, Conspiratifs, art, architecture, utopie, prospective

\section{AUTEUR}

\section{NOUHA BABAY}

Architecte d'intérieur, titulaire d'un master en sciences et techniques des arts (ISBAT, Tunis) et d'un master 2 recherche en histoire de l'architecture (université Panthéon-Sorbonne, 2015) sous la direction d'Éléonore Marantz nouha.babay@gmail.com 